\title{
Seesaw mirroring between light and heavy Majorana neutrinos with the help of the $S_{3}$ reflection symmetry
}

\author{
Zhi-Zhong Xing ${ }^{a, b}$ and Di Zhang ${ }^{a}$ \\ ${ }^{a}$ Theoretical Physics Division, Institute of High Energy Physics and School of Physical Sciences, \\ University of Chinese Academy of Sciences, \\ 19B Yuquan Road, Shijingshan District, Beijing 100049, China \\ ${ }^{b}$ Center for High Energy Physics, Peking University, \\ No. 5 Yiheyuan Road, Haidian District, Beijing 100080, China \\ E-mail: xingzz@ihep.ac.cn, zhangdi@ihep.ac.cn
}

ABSTRACT: In the canonical seesaw mechanism we require the relevant neutrino mass terms to be invariant under the $S_{3}$ charge-conjugation transformations of left- and right-handed neutrino fields. Then both the Dirac mass matrix $M_{\mathrm{D}}$ and the right-handed neutrino mass matrix $M_{\mathrm{R}}$ are well constrained, so is the effective light Majorana neutrino mass matrix $M_{\nu}$ via the seesaw formula. We find that these mass matrices can be classified into 22 categories, among which some textures respect the well-known $\mu-\tau$ permutation or reflection symmetry and flavor democracy. It is also found that there exist remarkable structural equalities or similarities between $M_{\nu}$ and $M_{\mathrm{R}}$, reflecting a seesaw mirroring relationship between light and heavy Majorana neutrinos. We calculate the corresponding light neutrino masses and flavor mixing parameters as well as the CP-violating asymmetries in decays of the lightest heavy Majorana neutrino, and show that only the flavored leptogenesis mechanism is possible to work for three categories of $M_{\mathrm{D}}$ and $M_{\mathrm{R}}$ in the $S_{3}$ reflection symmetry limit.

Keywords: Beyond Standard Model, Discrete Symmetries, Neutrino Physics, CP violation

ARXIV EPRINT: 1901.07912 


\section{Contents}

1 Introduction $\quad 1$

2 Applications of the $S_{3}$ reflection symmetry 5

2.1 Textures of $M_{\mathrm{D}}$ and $M_{\mathrm{R}}$ under $S_{3}$ reflection symmetry 5

2.2 The seesaw mirroring structure of $M_{\nu} \quad 8$

3 Neutrino masses and flavor mixing patterns $\quad \mathbf{1 2}$

$\begin{array}{lll}3.1 \text { Categories } A_{i} & 13\end{array}$

$\begin{array}{lll}3.2 & \text { Categories } B_{i} & 15\end{array}$

$\begin{array}{lll}3.3 & \text { Categories } C \text { and } D & 16\end{array}$

$\begin{array}{lll}3.4 & \text { Categories } E_{i} & 17\end{array}$

$\begin{array}{lll}3.5 & \text { Category } F & 17\end{array}$

$\begin{array}{lll}3.6 & \text { Categories } H_{i} & 18\end{array}$

$\begin{array}{lll}3.7 & \text { Categories } I_{i}, J \text { and } K & 19\end{array}$

4 Leptogenesis in the $S_{3}$ symmetry limit $\quad 20$

$\begin{array}{lll}4.1 & \text { Unflavored leptogenesis } & 21\end{array}$

4.1.1 Categories $A_{i} \quad 21$

$\begin{array}{lll}\text { 4.1.2 Category } C & 23\end{array}$

$\begin{array}{lll}4.2 & \text { Flavored leptogenesis } & 24\end{array}$

5 Some further discussions $\quad 27$

\section{Introduction}

The experimental discoveries of neutrino oscillations [1] have confessedly demonstrated that the standard model (SM) of particle physics is incomplete, because it cannot accommodate and explain the finite but tiny neutrino masses and significant lepton flavor mixing effects. The most canonical and popular way out is to introduce three right-handed neutrino fields $N_{\alpha \mathrm{R}}$ (for $\alpha=e, \mu, \tau$ ) and allow lepton number violation [2-6], with which the Yukawa interaction and a Majorana neutrino mass term can be written as

$$
-\mathcal{L}_{0}=\overline{\ell_{\mathrm{L}}} Y_{\nu} \widetilde{H} N_{\mathrm{R}}+\frac{1}{2} \overline{N_{\mathrm{R}}^{\mathrm{c}}} M_{\mathrm{R}} N_{\mathrm{R}}+\text { h.c. },
$$

where $\widetilde{H} \equiv \mathrm{i} \sigma_{2} H^{*}$ with $H$ being the Higgs doublet of the SM, $\ell_{\mathrm{L}}$ denotes the left-handed lepton doublet column vector, $N_{\mathrm{R}}$ represents the right-handed neutrino column vector with the $N_{\alpha \mathrm{R}}$ components, and $N_{\mathrm{R}}^{\mathrm{c}} \equiv \mathcal{C}{\overline{N_{\mathrm{R}}}}^{\mathrm{T}}$ with $\mathrm{T}$ denoting the transpose and $\mathcal{C}$ being the 
charge-conjugation operator. After spontaneous electroweak symmetry breaking, eq. (1.1) becomes

$$
-\mathcal{L}_{\mathrm{m}}=\overline{\nu_{\mathrm{L}}} M_{\mathrm{D}} N_{\mathrm{R}}+\frac{1}{2} \overline{N_{\mathrm{R}}^{\mathrm{c}}} M_{\mathrm{R}} N_{\mathrm{R}}+\text { h.c. },
$$

where $M_{\mathrm{D}} \equiv Y_{\nu}\langle H\rangle$ with $\langle H\rangle \simeq 174 \mathrm{GeV}$ being the vacuum expectation value of the Higgs field. The scale of $M_{\mathrm{R}}$ can be much larger than $\langle H\rangle$ because the right-handed neutrino fields are the $\mathrm{SU}(2)_{\mathrm{L}} \times \mathrm{U}(1)_{\mathrm{Y}}$ singlets and thus have nothing to do with electroweak symmetry breaking. In this case one may integrate out the heavy degrees of freedom and then obtain an effective mass term for the three light neutrinos:

$$
-\mathcal{L}_{\nu}=\frac{1}{2} \overline{\nu_{L}} M_{\nu} \nu_{\mathrm{L}}^{\mathrm{c}}+\text { h.c. },
$$

where $\nu_{\mathrm{L}}^{\mathrm{c}} \equiv \mathcal{C}{\overline{\nu_{\mathrm{L}}}}^{\mathrm{T}}$ is defined, and $M_{\nu}=-M_{\mathrm{D}} M_{\mathrm{R}}^{-1} M_{\mathrm{D}}^{\mathrm{T}}$ is the well-known seesaw formula $[2-$ $6]$ in the leading-order approximation, which naturally attributes the smallness of the scale of $M_{\nu}$ to the largeness of the scale of $M_{\mathrm{R}}$ as compared with the value of $\langle H\rangle$.

While the above seesaw relation can qualitatively explain why the masses of three light Majorana neutrinos $m_{i}$ (i.e., the eigenvalues of $M_{\nu}$ ) are strongly suppressed in magnitude, it unfortunately has no quantitative prediction for the values of $m_{i}$ and flavor mixing parameters. To reduce the number of unknown degrees of freedom and thus enhance the predictability and testability of the seesaw mechanism, the structures of $M_{\mathrm{D}}$ and $M_{\mathrm{R}}$ need to be specified with either some empirical assumptions (e.g., texture zeros - see ref. [7] for a review) or certain flavor symmetries (e.g., $A_{4}$ and $S_{4}$ symmetries - see refs. [8-10] for recent reviews). Since the observed pattern of the $3 \times 3$ Pontecorvo-MakiNakagawa-Sakata (PMNS) neutrino mixing matrix $V[11,12]$ exhibits an approximate $\mu-\tau$ permutation symmetry (i.e., $\left|V_{\mu i}\right| \simeq\left|V_{\tau i}\right|$ for $i=1,2,3$ ), most of the larger flavor symmetry groups considered for the neutrino sector actually consist of a subgroup which allows $M_{\nu}$ to respect the $\mu-\tau$ flavor symmetry. ${ }^{1}$

Of course, building a realistic neutrino mass model based on a given flavor symmetry is highly nontrivial because it is usually imperative to introduce some hypothetical gauge-singlet scalar fields (i.e., the so-called flavon fields) and make use of their vacuum expectation values to partly fix the flavor structures of massive neutrinos and charged leptons. Hence the flavor symmetry breaking is typically associated with many unknown parameters which are normally put into a hidden dustbin in most of the model-building exercises, since these new parameters are experimentally unaccessible for the time being. The variety of such models makes it practically hard to judge which flavor symmetry is closer to the truth [14].

In this situation one may follow a purely phenomenological way to focus only on the mass terms of charged leptons and neutrinos and then constrain their textures by means of certain flavor symmetries, so as to predict an acceptable flavor mixing pattern which is consistent with current neutrino oscillation data [8-10, 13]. Although there is an obvious gap between such an approach and a real neutrino mass model, the former can be regarded as a necessary or instructive step towards the latter. Considering that the underlying

\footnotetext{
${ }^{1}$ For a review with extensive references see ref. [13].
} 
flavor symmetry is most likely to manifest itself at a high energy scale far above the electroweak scale, the phenomenological approach under discussion actually fits the spirit of the bottom-up approach of model building in particle physics.

Following the same phenomenological approach, here we are interested in exploring the seesaw-induced relation between light and heavy Majorana neutrinos with the help of possible $S_{3}$ flavor symmetries. That is to say, we consider the possibility of simultaneously constraining the textures of $M_{\mathrm{D}}$ and $M_{\mathrm{R}}$ by requiring that $\mathcal{L}_{\mathrm{m}}$ in eq. (1.2) be invariant under the charge-conjugation transformations $\nu_{\mathrm{L}} \leftrightarrow \mathcal{S}_{(\mathrm{L})} \nu_{\mathrm{L}}^{\mathrm{c}}$ and $N_{\mathrm{R}} \leftrightarrow \mathcal{S}_{(\mathrm{R})} N_{\mathrm{R}}^{\mathrm{c}}$, where $\mathcal{S}_{(\mathrm{L})}$ or $\mathcal{S}_{(\mathrm{R})}$ stands for an arbitrary element belonging to an arbitrary subset of $S_{3}$ group. In this way it is easy to show that such a phenomenological requirement is equivalent to the constraints $M_{\mathrm{D}}^{*}=\mathcal{S}_{(\mathrm{L})}^{\dagger} M_{\mathrm{D}} \mathcal{S}_{(\mathrm{R})}$ and $M_{\mathrm{R}}^{*}=\mathcal{S}_{(\mathrm{R})} M_{\mathrm{R}} \mathcal{S}_{(\mathrm{R})}$, and therefore the structures of $M_{\mathrm{D}}$ and $M_{\mathrm{R}}$ can be strongly constrained. As a result, the structure of the light Majorana neutrino mass matrix $M_{\nu}$ can be partly determined via the seesaw formula $M_{\nu}=-M_{\mathrm{D}} M_{\mathrm{R}}^{-1} M_{\mathrm{D}}^{\mathrm{T}}$, leading to some intriguing predictions for the neutrino masses and flavor mixing parameters. In comparison with the so-called $\mu-\tau$ reflection symmetry which has been used to directly constrain the form of $M_{\nu}$ [15], our present method can be referred to as the $S_{3}$ reflection symmetry approach. Moreover, we find that the obtained texture of $M_{\nu}$ is either the same as or very similar to that of $M_{\mathrm{R}}$, a remarkable consequence of our approach which is referred to as the seesaw mirroring relationship between $M_{\nu}$ and $M_{\mathrm{R}}$. Along this line of thought, we also examine which of the $S_{3}$-constrained textures of $M_{\mathrm{D}}$ and $M_{\mathrm{R}}$ can allow for CP violation in the lepton-number-violating decays of the lightest heavy Majorana neutrino, a necessary ingredient of the thermal leptogenesis mechanism [16] which offers a natural explanation of the observed baryon asymmetry of the Universe.

It is worth pointing out that the $S_{3}$ reflection symmetry approach under discussion is subject to the basis with the flavor eigenstates of three charged leptons being the same as their mass eigenstates (i.e., the charged-lepton mass matrix $M_{l}$ is diagonal). Such a basis choice is different from the conventional model building exercises with the help of discrete flavor symmetries, in which the charged-lepton fields usually transform together with the neutrino fields under the given flavor groups [8-10]. After spontaneous flavor symmetry breaking, the charged-lepton and neutrino mass matrices are left with different residual symmetries. The basis with $M_{l}$ being diagonal can always be achieved by choosing a suitable representation of the given symmetry group, but it might not be convenient from the point of view of model building. In the present work we simply assume $M_{l}$ to be diagonal and make the $S_{3}$ reflection transformations only for the neutrino sector. This simple treatment allows us to directly derive the PMNS neutrino mixing matrix from the effective Majorana neutrino mass matrix $M_{\nu}$ via the seesaw relation, with no concern about the charged-lepton sector.

The approach and main results of this paper are expected to be instructive and useful for broadening our horizons in building realistic neutrino mass models and understanding lepton flavor mixing and $\mathrm{CP}$ violation. In fact, a lot of attention has been paid to applying the $S_{3}$ flavor symmetry to the quark and lepton sectors since the pioneering work done in 1978 [17, 18], and in this connection remarkable progress was made in 1996 and 1998 to predict quite large solar and atmospheric neutrino mixing angles [19-21]. Although some 
attempts have been made in combining the seesaw mechanism and the $S_{3}$ flavor symmetry (see, e.g., refs. [22-30]), our present work is different from them in several aspects:

- We constrain the structures of $M_{\mathrm{D}}$ and $M_{\mathrm{R}}$ by dictating the two neutrino mass terms in eq. (1.2) to be invariant under the $S_{3}$ reflection (i.e., charge-conjugation) transformations $\nu_{\mathrm{L}} \leftrightarrow \mathcal{S}_{(\mathrm{L})} \nu_{\mathrm{L}}^{\mathrm{c}}$ and $N_{\mathrm{R}} \leftrightarrow \mathcal{S}_{(\mathrm{R})} N_{\mathrm{R}}^{\mathrm{c}}$ instead of the $S_{3}$ permutation transformations $\nu_{\mathrm{L}} \leftrightarrow \mathcal{S}_{(\mathrm{L})} \nu_{\mathrm{L}}$ and $N_{\mathrm{R}} \leftrightarrow \mathcal{S}_{(\mathrm{R})} N_{\mathrm{R}}$. Such a new treatment makes sense because it is fully consistent with the spirit of the $\mu$ - $\tau$ reflection symmetry - a special case of the $S_{3}$ reflection symmetry under discussion, in order to produce the experimentally favored results $\theta_{23}=\pi / 4$ and $\delta=3 \pi / 2$ for the PMNS matrix $V$ in its standard parametrization form [1]. In comparison, one will be left with $\delta=0$ in the limit of the flavor democracy [19-21] or $S_{3}$ permutation symmetry [31-40].

- We carry out a systematic analysis of all the possible textures of $M_{\mathrm{D}}$ and $M_{\mathrm{R}}$ constrained by the $S_{3}$ reflection symmetry, make a classification of them, and examine whether the resulting textures of $M_{\nu}$ are seesaw-invariant or share the same flavor symmetry with $M_{\mathrm{R}}$ and (or) $M_{\mathrm{D}}$. Our results can therefore provide a very useful reference for further model-building exercises.

- We calculate the light neutrino masses, flavor mixing angles and CP-violting phases for each texture of $M_{\nu}$ in the $S_{3}$ refection symmetry limit, and examine whether the CP-violating asymmetries in decays of the lightest heavy Majorana neutrino are vanishing or not in the same limit. We find that in this case only flavored leptogenesis [41-43] is possible to work for a few textures of $M_{\mathrm{D}}$ and $M_{\mathrm{R}}$.

As many other flavor symmetries, the $S_{3}$ reflection symmetry must be broken too, so as to make $M_{\nu}$ fully fit current experimental data. One may discuss such symmetry breaking effects by either taking account of the renormalization-group evolution of $M_{\nu}$ from the seesaw scale (where the flavor symmetry is assumed to manifest itself) to the electroweak scale, or introducing some explicit symmetry breaking terms into $M_{\mathrm{D}}$ and $M_{\mathrm{R}}$ [13]. A further work of this kind depends on more technical details and empirical assumptions, and hence it is beyond the scope of the present paper and will be done elsewhere as a follow-up.

The remaining parts of this paper are organized as follows. In section 2 we first introduce the $S_{3}$ reflection transformations for left- and right-handed neutrino fields to constrain the structures of $M_{\mathrm{D}}$ and $M_{\mathrm{R}}$, and then determine the texture of $M_{\nu}$ with the help of the seesaw formula. All the possibilities in this connection are examined and classified. Section 3 is devoted to discussing the phenomenological consequences of $M_{\nu}$, where the light neutrino masses, flavor mixing angles and CP-violating phases are calculated in a case-bycase way. In section 4 we consider both unflavored and flavored leptogenesis mechanisms and calculate the corresponding CP-violating asymmetries in decays of the lightest heavy Majorana neutrino in the $S_{3}$ reflection symmetry limit. A summary of our approach and main results, together with some discussions about extending $S_{3}$ group to $A_{4}$ group so as to illustrate the constrained textures of neutrino mass matrices in a different way, is made in section 5 . 


\section{Applications of the $S_{3}$ reflection symmetry}

\subsection{Textures of $M_{\mathrm{D}}$ and $M_{\mathrm{R}}$ under $S_{3}$ reflection symmetry}

To begin with, we rewrite the mass terms in eq. (1.2) in a more concise way as follows:

$$
\left.-\mathcal{L}_{\mathrm{m}}=\frac{1}{2} \overline{\left(\nu_{\mathrm{L}} N_{\mathrm{R}}^{\mathrm{c}}\right.}\right)\left(\begin{array}{cc}
\mathbf{0} & M_{\mathrm{D}} \\
M_{\mathrm{D}}^{\mathrm{T}} & M_{\mathrm{R}}
\end{array}\right)\left(\begin{array}{c}
\nu_{\mathrm{L}}^{\mathrm{c}} \\
N_{\mathrm{R}}
\end{array}\right)+\text { h.c. . }
$$

To constrain flavor structures of the canonical seesaw mechanism, we require the neutrino mass term in eq. (2.1) to keep invariant when $\nu_{\mathrm{L}}$ and $N_{\mathrm{R}}$ transform as

$$
\nu_{\mathrm{L}} \leftrightarrow \mathcal{S}_{(\mathrm{L})} \nu_{\mathrm{L}}^{\mathrm{c}}, \quad N_{\mathrm{R}} \leftrightarrow \mathcal{S}_{(\mathrm{R})} N_{\mathrm{R}}^{\mathrm{c}}
$$

in which $\mathcal{S}_{\mathrm{L}}$ or $\mathcal{S}_{\mathrm{R}}$ denotes an arbitrary element of $G$ - a given subset of $S_{3}$ group, and the possibilities of both $\mathcal{S}_{\mathrm{L}}=\mathcal{S}_{\mathrm{R}}$ and $\mathcal{S}_{\mathrm{L}} \neq \mathcal{S}_{\mathrm{R}}$ are included. It is worth pointing out that only the neutrino mass term $\mathcal{L}_{\mathrm{m}}$ is dictated to be invariant under the transformations made in eq. (2.2), and hence the consequent $S_{3}$ reflection symmetry is not a real flavor symmetry for the whole Lagrangian of weak interactions. Instead, it only works as an effective organizing principle to simplify and constrain the structures of $M_{\mathrm{D}}$ and $M_{\mathrm{R}}$. Note that the well-known $\mu-\tau$ reflection symmetry and some other working flavor symmetries [10, 13] were proposed in the same spirit. If such a purely phenomenological approach turns out to be compatible with current and future experimental data, it may finally be embedded into a complete flavor model of fermion masses based on a larger symmetry group.

Under the $S_{3}$ reflection transformations given in eq. (2.2), the $6 \times 6$ neutrino mass matrix in eq. (2.1) changes as follows:

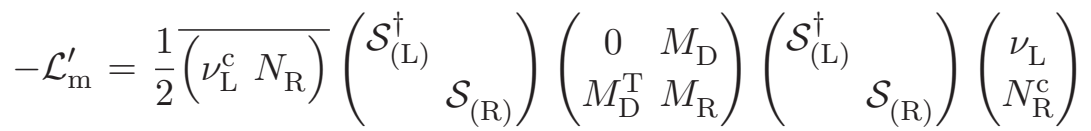

$$
\begin{aligned}
& +\frac{1}{2} \overline{\left(\begin{array}{ll}
\nu_{\mathrm{L}} & N_{\mathrm{R}}^{\mathrm{c}}
\end{array}\right)}\left(\begin{array}{cc}
\mathcal{S}_{(\mathrm{L})} & \\
& \mathcal{S}_{(\mathrm{R})}^{\dagger}
\end{array}\right)\left(\begin{array}{cc}
0 & M_{\mathrm{D}}^{*} \\
M_{\mathrm{D}}^{\dagger} & M_{\mathrm{R}}^{\dagger}
\end{array}\right)\left(\begin{array}{cc}
\mathcal{S}_{(\mathrm{L})} & \\
& \mathcal{S}_{(\mathrm{R})}^{\dagger}
\end{array}\right)\left(\begin{array}{c}
\nu_{\mathrm{L}}^{\mathrm{c}} \\
N_{\mathrm{R}}
\end{array}\right),
\end{aligned}
$$

where the unitarity of $\mathcal{S}_{(\mathrm{L})}$ and $\mathcal{S}_{(\mathrm{R})}$ has been used. It becomes transparent that the neutrino mass terms will be invariant (namely, $\mathcal{L}_{\mathrm{m}}=\mathcal{L}_{\mathrm{m}}^{\prime}$ ) if the whole neutrino mass matrix satisfies the condition

$$
\left(\begin{array}{cc}
0 & M_{\mathrm{D}} \\
M_{\mathrm{D}}^{\mathrm{T}} & M_{\mathrm{R}}
\end{array}\right)=\left(\begin{array}{cc}
0 & \mathcal{S}_{(\mathrm{L})} M_{\mathrm{D}}^{*} \mathcal{S}_{(\mathrm{R})}^{\dagger} \\
\mathcal{S}_{(\mathrm{R})}^{\dagger} M_{\mathrm{D}}^{\dagger} \mathcal{S}_{(\mathrm{L})} & \mathcal{S}_{(\mathrm{R})}^{\dagger} M_{\mathrm{R}}^{\dagger} \mathcal{S}_{(\mathrm{R})}^{\dagger}
\end{array}\right) .
$$

This in turn means that $M_{\mathrm{D}}$ and $M_{\mathrm{R}}$ should satisfy the conditions

$$
M_{\mathrm{D}}=\mathcal{S}_{(\mathrm{L})} M_{\mathrm{D}}^{*} \mathcal{S}_{(\mathrm{R})}^{\dagger}, \quad M_{\mathrm{R}}=\mathcal{S}_{(\mathrm{R})}^{\dagger} M_{\mathrm{R}}^{*} \mathcal{S}_{(\mathrm{R})}^{\dagger} .
$$

Therefore, the $S_{3}$ reflection symmetry imposed on the neutrino mass terms in eq. (2.1) allows us to constrain textures of the neutrino mass matrices $M_{\mathrm{D}}$ and $M_{\mathrm{R}}$. Such a constraint can be further transferred to the effective neutrino mass matrix $M_{\nu}$ via the seesaw formula

$$
M_{\nu}=-M_{\mathrm{D}} M_{\mathrm{R}}^{-1} M_{\mathrm{D}}^{\mathrm{T}},
$$


from which one may examine the structural similarity between $M_{\nu}$ and $M_{\mathrm{R}}$. In other words, it is possible to establish a seesaw mirroring relationship between light and heavy Majorana neutrinos with the help of the $S_{3}$ reflection symmetry.

Explicitly, the three-dimensional unitary representations for six elements of $S_{3}$ group are

$$
\begin{array}{rlrl}
S^{(123)} & =\left(\begin{array}{lll}
1 & 0 & 0 \\
0 & 1 & 0 \\
0 & 0 & 1
\end{array}\right), & S^{(231)}=\left(\begin{array}{lll}
0 & 1 & 0 \\
0 & 0 & 1 \\
1 & 0 & 0
\end{array}\right), & S^{(312)}=\left(\begin{array}{lll}
0 & 0 & 1 \\
1 & 0 & 0 \\
0 & 1 & 0
\end{array}\right), \\
S^{(213)}=\left(\begin{array}{lll}
0 & 1 & 0 \\
1 & 0 & 0 \\
0 & 0 & 1
\end{array}\right), & S^{(132)}=\left(\begin{array}{lll}
1 & 0 & 0 \\
0 & 0 & 1 \\
0 & 1 & 0
\end{array}\right), & S^{(321)}=\left(\begin{array}{lll}
0 & 0 & 1 \\
0 & 1 & 0 \\
1 & 0 & 0
\end{array}\right) .
\end{array}
$$

These group elements can be categorized into three conjugacy classes: $\mathcal{C}_{0}=\left\{S^{(123)}\right\}$, $\mathcal{C}_{1}=\left\{S^{(231)}, S^{(312)}\right\}$ and $\mathcal{C}_{2}=\left\{S^{(213)}, S^{(132)}, S^{(321)}\right\}$. So $S_{3}$ has one subgroup of order three, $Z_{3}=\left\{S^{(123)}, S^{(231)}, S^{(312)}\right\}$, as well as three subgroups of order two, $Z_{2}^{(12)}=$ $\left\{S^{(123)}, S^{(213)}\right\}, Z_{2}^{(23)}=\left\{S^{(123)}, S^{(132)}\right\}$ and $Z_{2}^{(31)}=\left\{S^{(123)}, S^{(321)}\right\}$. Note that $S_{3}$ group totally has $2^{3 !}-1=63$ non-void subsets. To characterize these subsets, we first reorder the elements of $S_{3}$ group as

$$
G^{6}=\left\{S^{(123)}, S^{(231)}, S^{(312)}, S^{(213)}, S^{(132)}, S^{(321)}\right\}
$$

Then an arbitrary subset can be characterized by $G_{i_{1} i_{2} \cdots i_{n}}^{n}$ or $G_{i_{1} i_{2} \cdots i_{6-n}}^{n}($ for $n=1,2, \cdots, 6)$, in which $n$ is the number of elements in the subset, and $i_{1} i_{2} \cdots i_{n}$ for $n \leq 3$ or $\overline{i_{1} i_{2} \cdots i_{6-n}}$ for $n>3$ is the index of different subsets with the equal number of elements. What is more, $i_{1}, i_{2}, \cdots, i_{n}=1,2, \cdots, 6$ should satisfy $i_{1}<i_{2}<\cdots<i_{n}$. Note that we are making use of the index $i_{1} i_{2} \cdots i_{n}$ for $n \leq 3$ which is a reordered sequence of the order numbers of elements belonging to $G_{i_{1} i_{2} \cdots i_{n}}^{n}$ in $G^{6}$, and the index $\overline{i_{1} i_{2} \cdots i_{6-n}}$ is used for $n>3$ which is a reordered sequence of the order numbers of elements belonging to the complement of $G_{i_{1} i_{2} \cdots i_{6-n}}^{n}$ with respect to $S_{3}$ group in $G^{6}$. For illustration, let us give several examples to make the notation issue clear: $G_{2}^{1}=\left\{S^{(231)}\right\}, G_{24}^{2}=\left\{S^{(231)}, S^{(213)}\right\}, G_{124}^{3}=\left\{S^{(123)}, S^{(231)}, S^{(213)}\right\}, G_{36}^{4}=$ $\left\{S^{(123)}, S^{(231)}, S^{(213)}, S^{(132)}\right\}$ and $G_{\overline{3}}^{5}=\left\{S^{(123)}, S^{(231)}, S^{(213)}, S^{(132)}, S^{(321)}\right\}$. It should also be noted that $G_{123}^{3}=Z_{3}, G_{14}^{2}=Z_{2}^{(12)}, G_{15}^{2}=Z_{2}^{(23)}$ and $G_{16}^{2}=Z_{2}^{(31)}$, and there are totally $C_{6}^{n}=\frac{6 !}{n !(6-n) !}$ different subsets $G_{i_{1} i_{2} \cdots i_{n}}^{n}$ or $G_{i_{1} i_{2} \cdots i_{6-n}}^{n}$ for a given number $n$.

With the help of eq. (2.5), we can now obtain all the possible structures of $M_{\mathrm{D}}$ and $M_{\mathrm{R}}$ constrained by the subsets of $S_{3}$ group. Here we take set $G_{5}^{1}$, corresponding to $\mathcal{S}_{(\mathrm{L})}=$ $\mathcal{S}_{(\mathrm{R})}=S^{(132)}$, as a typical example to do some explicit calculations. This case is particularly interesting because it actually works like the $\mu-\tau$ reflection symmetry. Since set $G_{5}^{1}$ contains 
only a single element (i.e., $S^{(132)}$ ), the mass matrices $M_{\mathrm{D}}$ and $M_{\mathrm{R}}$ satisfy

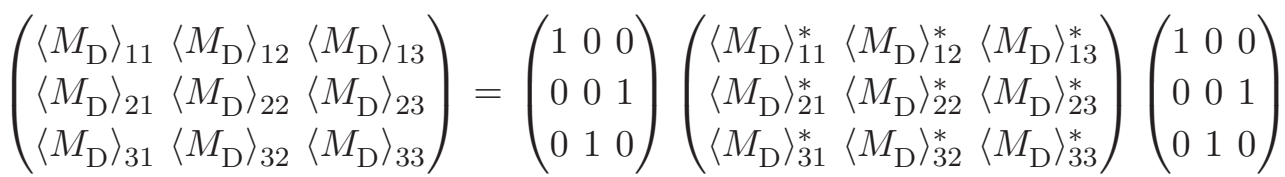

$$
\begin{aligned}
& =\left(\begin{array}{ccc}
\left\langle M_{\mathrm{D}}\right\rangle_{11}^{*} & \left\langle M_{\mathrm{D}}\right\rangle_{13}^{*} & \left\langle M_{\mathrm{D}}\right\rangle_{12}^{*} \\
\left\langle M_{\mathrm{D}}\right\rangle_{31}^{*} & \left\langle M_{\mathrm{D}}\right\rangle_{33}^{*} & \left\langle M_{\mathrm{D}}\right\rangle_{32}^{*} \\
\left\langle M_{\mathrm{D}}\right\rangle_{21}^{*} & \left\langle M_{\mathrm{D}}\right\rangle_{23}^{*} & \left\langle M_{\mathrm{D}}\right\rangle_{22}^{*}
\end{array}\right)
\end{aligned}
$$

and

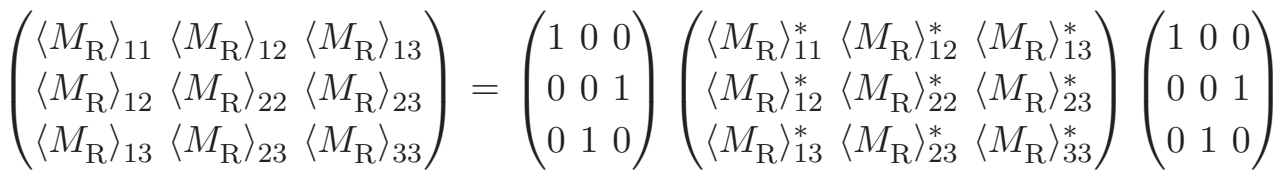

$$
\begin{aligned}
& =\left(\begin{array}{ccc}
\left\langle M_{\mathrm{R}}\right\rangle_{11}^{*} & \left\langle M_{\mathrm{R}}\right\rangle_{13}^{*} & \left\langle M_{\mathrm{R}}\right\rangle_{12}^{*} \\
\left\langle M_{\mathrm{R}}\right\rangle_{13}^{*} & \left\langle M_{\mathrm{R}}\right\rangle_{33}^{*} & \left\langle M_{\mathrm{R}}\right\rangle_{23}^{*} \\
\left\langle M_{\mathrm{R}}\right\rangle_{12}^{*} & \left\langle M_{\mathrm{R}}\right\rangle_{23}^{*} & \left\langle M_{\mathrm{R}}\right\rangle_{22}^{*}
\end{array}\right) .
\end{aligned}
$$

As a result, we arrive at

$$
\begin{aligned}
& \left\langle M_{\mathrm{D}}\right\rangle_{11}=\left\langle M_{\mathrm{D}}\right\rangle_{11}^{*}, \quad\left\langle M_{\mathrm{D}}\right\rangle_{12}=\left\langle M_{\mathrm{D}}\right\rangle_{13}^{*}, \\
& \left\langle M_{\mathrm{D}}\right\rangle_{21}=\left\langle M_{\mathrm{D}}\right\rangle_{31}^{*}, \quad\left\langle M_{\mathrm{D}}\right\rangle_{22}=\left\langle M_{\mathrm{D}}\right\rangle_{33}^{*}, \\
& \left\langle M_{\mathrm{D}}\right\rangle_{23}=\left\langle M_{\mathrm{D}}\right\rangle_{32}^{*} ;
\end{aligned}
$$

and

$$
\begin{aligned}
& \left\langle M_{\mathrm{R}}\right\rangle_{11}=\left\langle M_{\mathrm{R}}\right\rangle_{11}^{*}, \quad\left\langle M_{\mathrm{R}}\right\rangle_{12}=\left\langle M_{\mathrm{R}}\right\rangle_{13}^{*}, \\
& \left\langle M_{\mathrm{R}}\right\rangle_{22}=\left\langle M_{\mathrm{R}}\right\rangle_{33}^{*}, \quad\left\langle M_{\mathrm{R}}\right\rangle_{23}=\left\langle M_{\mathrm{R}}\right\rangle_{23}^{*} \text {. }
\end{aligned}
$$

According to eqs. (2.11) and (2.12), the textures of $M_{\mathrm{D}}$ and $M_{\mathrm{R}}$ can be parametrized as

$$
M_{\mathrm{D}}=\left(\begin{array}{ccc}
A_{\mathrm{r}} & B & B^{*} \\
E & C & D \\
E^{*} & D^{*} & C^{*}
\end{array}\right), \quad M_{\mathrm{R}}=\left(\begin{array}{ccc}
a_{\mathrm{r}} & b & b^{*} \\
b & e & d_{\mathrm{r}} \\
b^{*} & d_{\mathrm{r}} & e^{*}
\end{array}\right)
$$

where the subscript " $\mathrm{r}$ " means that this element is real. Taking account of the seesaw formula in eq. (2.6), we find that the effective neutrino mass matrix $M_{\nu}$ has the following texture:

$$
M_{\nu}=\left(\begin{array}{ccc}
a_{\mathrm{r}}^{\prime} & b^{\prime} & b^{*} \\
b^{\prime} & e^{\prime} & d_{\mathrm{r}}^{\prime} \\
b^{\prime *} & d_{\mathrm{r}}^{\prime} & e^{\prime *}
\end{array}\right)
$$


where $a_{\mathrm{r}}^{\prime}, b^{\prime}, d_{\mathrm{r}}^{\prime}$ and $e^{\prime}$ are explicitly given by

$$
\begin{aligned}
\begin{aligned}
& a_{\mathrm{r}}^{\prime}=-\frac{1}{\operatorname{det} M_{\mathrm{R}}}\{ A_{\mathrm{r}}^{2}\left(|e|^{2}-d_{\mathrm{r}}^{2}\right)+4 A_{\mathrm{r}} \operatorname{Re}\left[B\left(d_{\mathrm{r}} b^{*}-b e^{*}\right)\right]+2 \operatorname{Re}\left[B^{2}\left(a_{\mathrm{r}} e^{*}-b^{* 2}\right)\right] \\
&\left.+2|B|^{2}\left(|b|^{2}-a_{\mathrm{r}} d\right)\right\}, \\
& b^{\prime}=-\frac{1}{\operatorname{det} M_{\mathrm{R}}}\{ A_{\mathrm{r}} E\left(|e|^{2}-d_{\mathrm{r}}^{2}\right)+2 E \operatorname{Re}\left[B\left(d_{\mathrm{r}} b^{*}-b e^{*}\right)\right]+A_{\mathrm{r}} C\left(d_{\mathrm{r}} b^{*}-b e^{*}\right) \\
&+B C\left(a_{\mathrm{r}} e^{*}-b^{* 2}\right)+\left(B^{*} C+B D\right)\left(|b|^{2}-a d_{\mathrm{r}}\right)+A_{\mathrm{r}} D\left(b d_{\mathrm{r}}-b^{*} e\right) \\
&\left.+B^{*} D\left(a_{\mathrm{r}} e-b^{2}\right)\right\}, \\
& e^{\prime}=-\frac{1}{\operatorname{det} M_{\mathrm{R}}}[ E^{2}\left(|e|^{2}-d_{\mathrm{r}}^{2}\right)+2 E C\left(d_{\mathrm{r}} b^{*}-b e^{*}\right)+2 E D\left(b d_{\mathrm{r}}-b^{*} e\right) \\
&\left.+C^{2}\left(a_{\mathrm{r}} e^{*}-b^{* 2}\right)+2 C D\left(|b|^{2}-a_{\mathrm{r}} d_{\mathrm{r}}\right)+D^{2}\left(a_{\mathrm{r}} e-b^{2}\right)\right], \\
& d_{\mathrm{r}}^{\prime}=-\frac{1}{\operatorname{det} M_{\mathrm{R}}}\left\{|E|^{2}\left(|e|^{2}-d_{\mathrm{r}}^{2}\right)+2 \operatorname{Re}\left[\left(E^{*} C+E D^{*}\right)\left(d_{\mathrm{r}} b^{*}-b e^{*}\right)\right]\right. \\
&\left.+\left(|C|^{2}+|D|^{2}\right)\left(|b|^{2}-a_{\mathrm{r}} d_{\mathrm{r}}\right)+2 \operatorname{Re}\left[C D^{*}\left(a_{\mathrm{r}} e^{*}-b^{* 2}\right)\right]\right\},
\end{aligned}
\end{aligned}
$$

with $\operatorname{det} M_{\mathrm{R}}=a_{\mathrm{r}}|e|^{2}+2|b|^{2} d_{\mathrm{r}}-a_{\mathrm{r}} d_{\mathrm{r}}^{2}-2 \operatorname{Re}\left(b^{2} e^{*}\right)$. We see that $M_{\nu}$ and $M_{\mathrm{R}}$ have the same structure respecting the $\mu-\tau$ reflection symmetry, and therefore there exists an interesting seesaw mirroring relationship between light and heavy Majorana neutrinos.

The other possibilities can be similarly discussed by repeating the above procedure with either sets $G^{n}$ corresponding to $\mathcal{S}_{(\mathrm{L})}=\mathcal{S}_{(\mathrm{R})}$ or sets $G_{\mathrm{L}}^{n} \times G_{\mathrm{R}}^{n}$ including both $\mathcal{S}_{(\mathrm{L})}=\mathcal{S}_{(\mathrm{R})}$ and $\mathcal{S}_{(\mathrm{L})} \neq \mathcal{S}_{(\mathrm{R})}$ options. In table 1 we list and classify the textures of $M_{\mathrm{D}}, M_{\mathrm{R}}$ and $M_{\nu}$ corresponding to all the possible sets under consideration. For the sake of simplicity, the explicit relations between the parameters of $M_{\nu}$ and those of $M_{\mathrm{D}}$ and $M_{\mathrm{R}}$ have been omitted from table 1 .

\subsection{The seesaw mirroring structure of $M_{\nu}$}

Table 1 provides a classification of all the possible structures of $M_{\nu}$ in accordance with those of $M_{\mathrm{D}}$ and $M_{\mathrm{R}}$. For each category of $M_{\nu}$, its structure is the same as or similar to the structure of $M_{\mathrm{R}}$ or $M_{\mathrm{D}}$, reflecting the seesaw mirroring feature that we have stressed.

The classification is certainly based on eqs. (2.5) and (2.6). If the textures of $M_{\mathrm{D}}$, $M_{\mathrm{R}}$ and $M_{\nu}$ constrained by different sets, such as $G_{i_{1} i_{2} \cdots i_{n}}^{n}\left(\right.$ or $\left.G_{i_{1} i_{2} \cdots i_{n} \mathrm{~L}}^{n} \times G_{i_{1} i_{2} \cdots i_{n} \mathrm{R}}^{n}\right)$ or

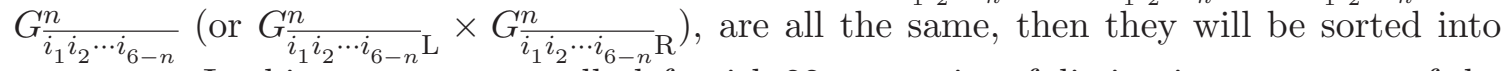
one group. In this way we are totally left with 22 categories of distinctive structures of the mass matrices, as listed in table 1 . Note that the mass matrices belonging to categories $A_{1}, A_{2}$ and $A_{3}$ are actually correlated with each other via a transformation associated with $S^{(231)}$ and $S^{(312)}$. To be specific,

$$
\begin{array}{ll}
M_{\mathrm{D}}^{\left(A_{2}\right)}=S^{(231)} M_{\mathrm{D}}^{\left(A_{1}\right)} S^{(312)}, & M_{\mathrm{D}}^{\left(A_{3}\right)}=S^{(312)} M_{\mathrm{D}}^{\left(A_{1}\right)} S^{(231)}, \\
M_{\mathrm{R}}^{\left(A_{2}\right)}=S^{(231)} M_{\mathrm{R}}^{\left(A_{1}\right)} S^{(312)}, & M_{\mathrm{R}}^{\left(A_{3}\right)}=S^{(312)} M_{\mathrm{R}}^{\left(A_{1}\right)} S^{(231)}, \\
M_{\nu}^{\left(A_{2}\right)}=S^{(231)} M_{\nu}^{\left(A_{1}\right)} S^{(312)}, & M_{\nu}^{\left(A_{3}\right)}=S^{(312)} M_{\nu}^{\left(A_{1}\right)} S^{(231)} .
\end{array}
$$




\begin{tabular}{|c|c|c|c|c|}
\hline Cases & $M_{\mathrm{D}}$ & $M_{\mathrm{R}}$ & $M_{\nu}$ & Sets \\
\hline & $\left(\begin{array}{lll}A_{\mathrm{r}} & B & B^{*}\end{array}\right.$ & $\begin{array}{lll}a_{\mathrm{r}} & b & b^{*}\end{array}$ & $\begin{array}{lll}a_{\mathrm{r}}^{\prime} & b^{\prime} & b^{\prime *} \\
\end{array}$ & \\
\hline$A_{1}$ & $\begin{array}{lll}E & C & D\end{array}$ & $\begin{array}{llll}b & e & d_{\mathrm{r}}\end{array}$ & $\begin{array}{lll}b^{\prime} & e^{\prime} & d_{\mathrm{r}}^{\prime}\end{array}$ & $G_{5}^{1}, G_{5 \mathrm{~L}}^{1} \times G_{5 \mathrm{R}}^{1}$ \\
\hline & $\left(E^{*} D^{*} C^{*}\right)$ & $\left(b^{*} d_{\mathrm{r}} e^{*}\right)$ & $b^{\prime *} d_{\mathrm{r}}^{\prime} e^{\prime *}$ & \\
\hline & $\left(\begin{array}{lll}C & D & E\end{array}\right)$ & $\left.\begin{array}{lll}e & d_{\mathrm{r}} & b\end{array}\right)$ & $\left(\begin{array}{lll}e^{\prime} & d_{\mathrm{r}}^{\prime} & b^{\prime}\end{array}\right)$ & \\
\hline$A_{2}$ & $D^{*} C^{*} E^{*}$ & $d_{\mathrm{r}} e^{*} b^{*}$ & $d_{\mathrm{r}}^{\prime} e^{\prime *} b^{\prime *}$ & $G_{4}^{1}, G_{4 \mathrm{~L}}^{1} \times G_{4 \mathrm{R}}^{1}$ \\
\hline & $\left(\begin{array}{lll}B & B^{*} & A_{\mathrm{r}}\end{array}\right)$ & $\left(\begin{array}{lll}b & b^{*} & a_{\mathrm{r}}\end{array}\right)$ & $\left(\begin{array}{lll}b^{\prime} & b^{\prime *} & a_{\mathrm{r}}^{\prime}\end{array}\right)$ & \\
\hline & $\left(C^{*} E^{*} D^{*}\right)$ & $\left(e^{*} b^{*} d_{\mathrm{r}}\right)$ & 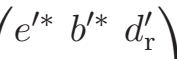 & \\
\hline$A_{3}$ & $B^{*} A_{\mathrm{r}} B$ & $b^{*} a_{\mathrm{r}} b$ & $b^{\prime *} \quad a_{\mathrm{r}}^{\prime} b^{\prime}$ & $G_{6}^{1}, G_{6 \mathrm{~L}}^{1} \times G_{6 \mathrm{R}}^{1}$ \\
\hline & $\left(\begin{array}{lll}D & E & C\end{array}\right)$ & $\left(\begin{array}{lll}d_{\mathrm{r}} & b & e\end{array}\right)$ & $\left(\begin{array}{lll}d_{\mathrm{r}}^{\prime} & b^{\prime} & e^{\prime}\end{array}\right)$ & \\
\hline & $\left(\begin{array}{lll}A_{\mathrm{r}} & B_{\mathrm{r}} & B_{\mathrm{r}}\end{array}\right)$ & $\left.a_{\mathrm{r}} b_{\mathrm{r}} b_{\mathrm{r}}\right)$ & $\left(\begin{array}{lll}a_{\mathrm{r}}^{\prime} & b_{\mathrm{r}}^{\prime} & b_{\mathrm{r}}^{\prime}\end{array}\right)$ & \\
\hline$B_{1}$ & $E_{\mathrm{r}} C_{\mathrm{r}} D_{\mathrm{r}}$ & $b_{\mathrm{r}} e_{\mathrm{r}} d_{\mathrm{r}}$ & $b_{\mathrm{r}}^{\prime} e_{\mathrm{r}}^{\prime} d_{\mathrm{r}}^{\prime}$ & $G_{15}^{2}$ \\
\hline & $\left(\begin{array}{lll}E_{\mathrm{r}} & D_{\mathrm{r}} & C_{\mathrm{r}}\end{array}\right)$ & $\left(\begin{array}{lll}b_{\mathrm{r}} & d_{\mathrm{r}} & e_{\mathrm{r}}\end{array}\right)$ & $\left(\begin{array}{lll}b_{\mathrm{r}}^{\prime} & d_{\mathrm{r}}^{\prime} & e_{\mathrm{r}}^{\prime}\end{array}\right)$ & \\
\hline & $\left(\begin{array}{lll}C_{\mathrm{r}} & D_{\mathrm{r}} & E_{\mathrm{r}}\end{array}\right)$ & $\left(e_{\mathrm{r}} d_{\mathrm{r}} b_{\mathrm{r}}\right)$ & $\left(\begin{array}{lll}e_{\mathrm{r}}^{\prime} & d_{\mathrm{r}}^{\prime} & b_{\mathrm{r}}^{\prime}\end{array}\right)$ & \\
\hline$B_{2}$ & $D_{\mathrm{r}} C_{\mathrm{r}} E_{\mathrm{r}}$ & $d_{\mathrm{r}} e_{\mathrm{r}} b_{\mathrm{r}}$ & $d_{\mathrm{r}}^{\prime} e_{\mathrm{r}}^{\prime} b_{\mathrm{r}}^{\prime}$ & $G_{14}^{2}$ \\
\hline & $\left(\begin{array}{lll}B_{\mathrm{r}} & B_{\mathrm{r}} & A_{\mathrm{r}}\end{array}\right)$ & $\left(\begin{array}{lll}b_{\mathrm{r}} & b_{\mathrm{r}} & a_{\mathrm{r}}\end{array}\right)$ & $\left(\begin{array}{lll}b_{\mathrm{r}}^{\prime} & b_{\mathrm{r}}^{\prime} & a_{\mathrm{r}}^{\prime}\end{array}\right)$ & \\
\hline & $\left(\begin{array}{lll}C_{\mathrm{r}} & E_{\mathrm{r}} & D_{\mathrm{r}}\end{array}\right)$ & $\left(\begin{array}{lll}e_{\mathrm{r}} & b_{\mathrm{r}} & d_{\mathrm{r}}\end{array}\right)$ & $\left(\begin{array}{lll}e_{\mathrm{r}}^{\prime} & b_{\mathrm{r}}^{\prime} & d_{\mathrm{r}}^{\prime}\end{array}\right)$ & \\
\hline$B_{3}$ & $B_{\mathrm{r}} A_{\mathrm{r}} B_{\mathrm{r}}$ & $b_{\mathrm{r}} a_{\mathrm{r}} b_{\mathrm{r}}$ & $b_{\mathrm{r}}^{\prime} a_{\mathrm{r}}^{\prime} b_{\mathrm{r}}^{\prime}$ & $G_{16}^{2}$ \\
\hline & $\left(\begin{array}{lll}D_{\mathrm{r}} & E_{\mathrm{r}} & C_{\mathrm{r}}\end{array}\right)$ & $\left(\begin{array}{lll}d_{\mathrm{r}} & b_{\mathrm{r}} & e_{\mathrm{r}}\end{array}\right)$ & $\left(\begin{array}{lll}d_{\mathrm{r}}^{\prime} & b_{\mathrm{r}}^{\prime} & e_{\mathrm{r}}^{\prime}\end{array}\right)$ & \\
\hline & $\left(\begin{array}{lll}A_{\mathrm{r}} & B & B^{*}\end{array}\right)$ & $\left.\begin{array}{lll}a_{\mathrm{r}} & b_{\mathrm{r}} & b_{\mathrm{r}}\end{array}\right)$ & $\left(\begin{array}{lll}a_{\mathrm{r}}^{\prime} & b_{\mathrm{r}}^{\prime} & b_{\mathrm{r}}^{\prime}\end{array}\right)$ & $G_{45}^{2}, G_{46}^{2}$ \\
\hline$C$ & $\begin{array}{ccc}B^{*} & A_{\mathrm{r}} & B \\
D & D^{*} & \end{array}$ & $b_{\mathrm{r}} a_{\mathrm{r}} b_{\mathrm{r}}$ & $\left.\begin{array}{lll}b_{\mathrm{r}}^{\prime} & a_{\mathrm{r}}^{\prime} & b_{\mathrm{r}}^{\prime} \\
h^{\prime} & h^{\prime} & a^{\prime}\end{array}\right)$ & $G_{56}^{2}, G_{456}^{3}$ \\
\hline & $\left(\begin{array}{lll}B & B^{*} & A_{\mathrm{r}}\end{array}\right)$ & $\left(\begin{array}{lll}b_{\mathrm{r}} & b_{\mathrm{r}} & a_{\mathrm{r}}\end{array}\right)$ & $\left(\begin{array}{lll}b_{\mathrm{r}}^{\prime} & b_{\mathrm{r}}^{\prime} & a_{\mathrm{r}}^{\prime}\end{array}\right)$ & \\
\hline$D$ & $\left(\begin{array}{ccc}A_{\mathrm{r}} & B_{\mathrm{r}} & B_{\mathrm{r}} \\
B_{\mathrm{r}} & A_{\mathrm{r}} & B_{\mathrm{r}}\end{array}\right)$ & $\left.\begin{array}{ccc}a_{\mathrm{r}} & b_{\mathrm{r}} & b_{\mathrm{r}} \\
b_{\mathrm{r}} & a_{\mathrm{r}} & b_{\mathrm{r}}\end{array}\right)$ & $\left(\begin{array}{ccc}a_{\mathrm{r}}^{\prime} & b_{\mathrm{r}}^{\prime} & b_{\mathrm{r}}^{\prime} \\
b_{\mathrm{r}}^{\prime} & a_{\mathrm{r}}^{\prime} & b_{\mathrm{r}}^{\prime}\end{array}\right)$ & $G_{145}^{3}, G_{146}^{3}$ \\
\hline & $\left(\begin{array}{lll}B_{\mathrm{r}} & B_{\mathrm{r}} & A_{\mathrm{r}}\end{array}\right)$ & $\left(\begin{array}{lll}b_{\mathrm{r}} & b_{\mathrm{r}} & a_{\mathrm{r}}\end{array}\right)$ & $\left(\begin{array}{lll}b_{\mathrm{r}}^{\prime} & b_{\mathrm{r}}^{\prime} & a_{\mathrm{r}}^{\prime}\end{array}\right)$ & $G_{156}^{3}, G_{23}^{4}$ \\
\hline & $\left(\begin{array}{lll}A_{\mathrm{r}} & B_{\mathrm{r}} & B_{\mathrm{r}}\end{array}\right)$ & $\left.a_{\mathrm{r}} b_{\mathrm{r}} b_{\mathrm{r}}\right)$ & $\left(\begin{array}{lll}a_{\mathrm{r}}^{\prime} & b_{\mathrm{r}}^{\prime} & b_{\mathrm{r}}^{\prime}\end{array}\right)$ & $G_{25}^{2}, G_{35}^{2}, G_{125}^{3}$ \\
\hline$E_{1}$ & $\left(\begin{array}{ccc}B_{\mathrm{r}} & A_{\mathrm{r}} & B_{\mathrm{r}} \\
B_{\mathrm{r}} & B_{\mathrm{r}} & A_{\mathrm{r}}\end{array}\right)$ & $\left.\begin{array}{lll}b_{\mathrm{r}} & b_{\mathrm{r}} & a_{\mathrm{r}} \\
b_{\mathrm{r}} & a_{\mathrm{r}} & b_{\mathrm{r}}\end{array}\right)$ & $\left(\begin{array}{lll}b_{\mathrm{r}}^{\prime} & b_{\mathrm{r}}^{\prime} & a_{\mathrm{r}}^{\prime} \\
b_{\mathrm{r}}^{\prime} & a_{\mathrm{r}}^{\prime} & b_{\mathrm{r}}^{\prime}\end{array}\right)$ & $G_{135}^{3}, G_{235}^{3}, G_{46}^{4}$ \\
\hline & $\left(\begin{array}{lll}A_{\mathrm{r}} & B_{\mathrm{r}} & B_{\mathrm{r}}\end{array}\right)$ & $\left(\begin{array}{lll}b_{\mathrm{r}} & a_{\mathrm{r}} & b_{\mathrm{r}}\end{array}\right)$ & $\left(\begin{array}{lll}b_{\mathrm{r}}^{\prime} & a_{\mathrm{r}}^{\prime} & b_{\mathrm{r}}^{\prime}\end{array}\right)$ & $G_{24}^{2}, G_{24}^{2}, G_{12}^{3}$ \\
\hline$E_{2}$ & $\left|\begin{array}{ccc}B_{\mathrm{r}} & A_{\mathrm{r}} & B_{\mathrm{r}} \\
B & B & A\end{array}\right|$ & 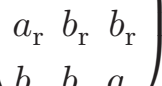 & $\left.\begin{array}{lll}a_{\mathrm{r}}^{\prime} & b_{\mathrm{r}}^{\prime} & b_{\mathrm{r}}^{\prime} \\
h^{\prime} & b^{\prime} & a^{\prime}\end{array}\right)$ & $G_{134}^{3}, G_{234}^{3}, G_{56}^{4}$ \\
\hline & $\left(\begin{array}{lll}A_{\mathrm{r}} & B_{\mathrm{r}} & B_{\mathrm{r}}\end{array}\right)$ & $\left(\begin{array}{lll}v_{\mathrm{r}} & \mathrm{r}_{\mathrm{r}} & a_{\mathrm{r}} \\
b_{\mathrm{r}} & b_{\mathrm{r}} & a_{\mathrm{r}}\end{array}\right)$ & $\left(\begin{array}{lll}b_{\mathrm{r}}^{\prime} & b_{\mathrm{r}}^{\prime} & a_{\mathrm{r}}^{\prime}\end{array}\right)$ & \\
\hline$E_{3}$ & $B_{\mathrm{r}} A_{\mathrm{r}} B_{\mathrm{r}}$ & $b_{\mathrm{r}} a_{\mathrm{r}} b_{\mathrm{r}}$ & $b_{\mathrm{r}}^{\prime} a_{\mathrm{r}}^{\prime} b_{\mathrm{r}}^{\prime}$ & $\begin{array}{r}G_{26}, G_{36}^{3}, G_{126} \\
G^{3}, G^{3}, G\end{array}$ \\
\hline & $\left(\begin{array}{lll}B_{\mathrm{r}} & B_{\mathrm{r}} & A_{\mathrm{r}}\end{array}\right)$ & $\left(\begin{array}{lll}a_{\mathrm{r}} & b_{\mathrm{r}} & b_{\mathrm{r}}\end{array}\right)$ & $\left(\begin{array}{lll}a_{\mathrm{r}}^{\prime} & b_{\mathrm{r}}^{\prime} & b_{\mathrm{r}}^{\prime}\end{array}\right)$ & $G_{136}, G_{236}, G_{45}$ \\
\hline
\end{tabular}

Table 1. All the possible structures of $M_{\mathrm{D}}$ and $M_{\mathrm{R}}$ constrained by sets $G^{n}$ or $G_{\mathrm{L}}^{n} \times G_{\mathrm{R}}^{n}$ in the $S_{3}$ reflection symmetry limit, and the consequent structures of $M_{\nu}$ via the seesaw formula. The subscript " $\mathrm{r}$ " of a given matrix element means that this element is real. 


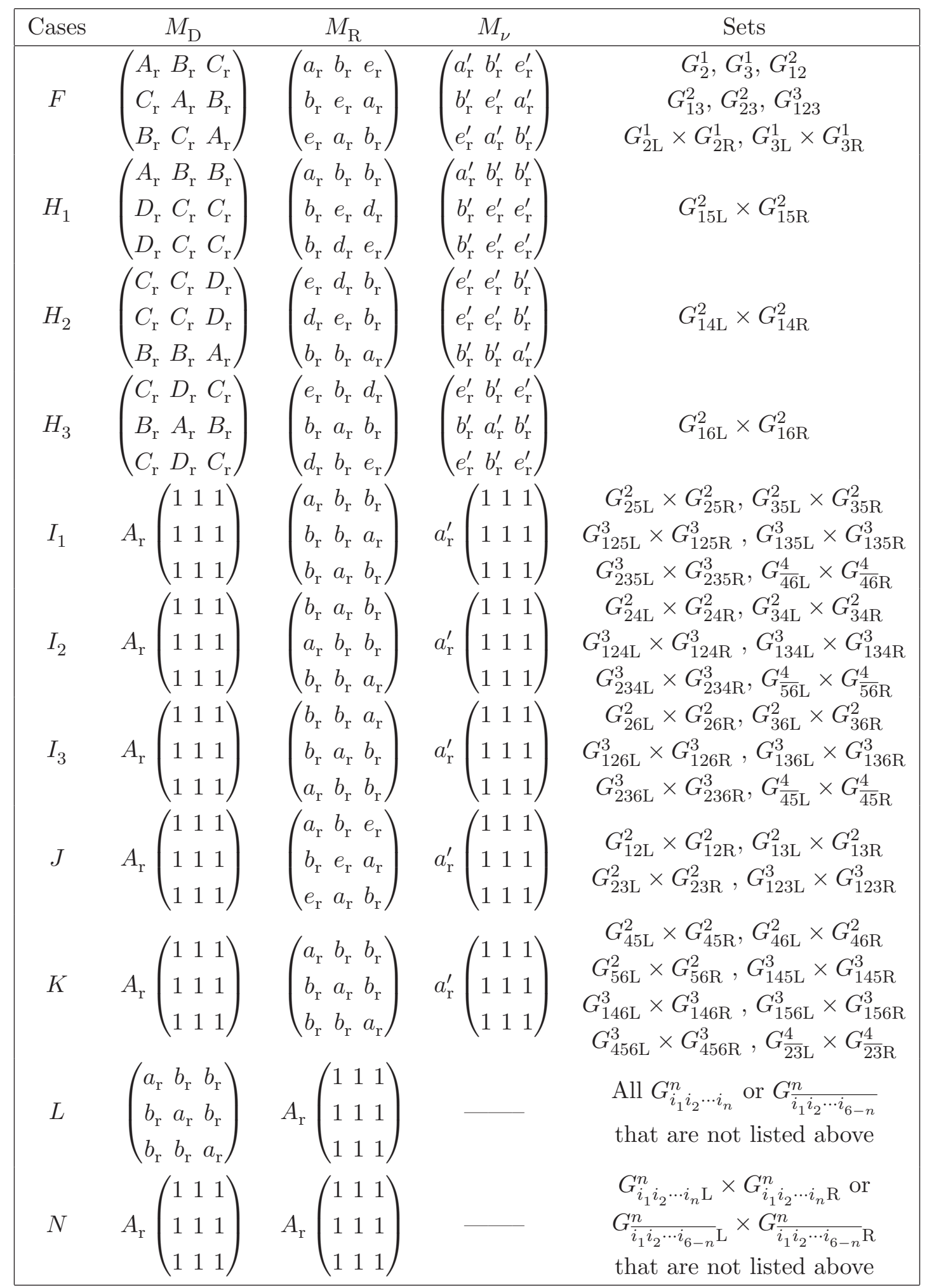

Table 1. Continued. 
We find that the same correlations exist for $M_{\mathrm{D}}, M_{\mathrm{R}}$ and $M_{\nu}$ in categories $B_{i}, E_{i}, H_{i}$ and $I_{i}$ (for $\left.i=1,2,3\right)$. In fact, eq. (2.16) for categories $A_{i}$ and similar relations of this kind for other categories can be understood as follows.

1. The corresponding sets in categories $X_{1}, X_{2}$ and $X_{3}$ (for $X=A, B, E, H$ or $I$ ) contain $S^{(132)}, S^{(213)}$ and $S^{(321)}$, respectively. The other possible elements (i.e., $S^{(123)}$, $\left.S^{(231)}, S^{(312)}\right)$ contained by $X_{1}$ are simultaneously contained by $X_{2}$ and $X_{3}$.

2. The three-dimensional representation of $S_{3}$ group in eq. (2.7) is a unitary representation, and hence $\mathcal{S}_{(\mathrm{L})}^{\dagger}=\mathcal{S}_{(\mathrm{L})}^{-1}$ and $\mathcal{S}_{(\mathrm{R})}^{\dagger}=\mathcal{S}_{(\mathrm{R})}^{-1}$ hold.

3. Since $S^{(132)}, S^{(213)}$ and $S^{(321)}$ belong to one conjugacy class $\mathcal{C}_{2}$, they can be connected with one another by one element of $S_{3}$ group. Namely, $S^{(213)}=S^{(231)} S^{(132)}\left[S^{(231)}\right]^{-1}$, $S^{(321)}=S^{(312)} S^{(132)}\left[S^{(312)}\right]^{-1}$ and $S^{(213)}=S^{(312)} S^{(321)}\left[S^{(312)}\right]^{-1}$.

4. The conjugacy class $\mathcal{C}_{1}$ containing elements $S^{(231)}$ and $S^{(312)}$ is a self reciprocal class, and the subgroup $Z_{3}$ is an Abelian group. As a result, $S^{(231)}=\left[S^{(312)}\right]^{-1}$, $S^{(312)}=\left[S^{(231)}\right]^{-1},\left[S^{(231)}\right]^{2}=S^{(312)}$ and $\left[S^{(312)}\right]^{2}=S^{(231)}$ hold, and the three elements of $Z_{3}$ commute with one another (i.e., $\left[S^{(123)}, S^{(231)}\right]=\left[S^{(123)}, S^{(312)}\right]=$ $\left.\left[S^{(312)}, S^{(231)}\right]=0\right)$.

These properties, together with eqs. (2.5) and (2.6), allow us to easily obtain eq. (2.16) and other similar relations. Such relations are very helpful in the sense that once the result for one case is achieved, the results for the other two cases can be conveniently figured out in no need of repeating the relevant calculations.

The notation $G_{\mathrm{L}}^{n} \times G_{\mathrm{R}}^{n}$ in table 1 means that the left-handed fields $\nu_{\mathrm{L}}$ and the righthanded fields $N_{\mathrm{R}}$ can transform with different elements of $G^{n}$, corresponding to $\mathcal{S}_{\mathrm{L}}$ for $\nu_{\mathrm{L}}$ and $\mathcal{S}_{\mathrm{R}}$ for $N_{\mathrm{R}}$ shown in eq. $(2.2)$, where $\mathcal{S}_{\mathrm{L}}$ and $\mathcal{S}_{\mathrm{R}}$ can be either identical or different. ${ }^{2}$ Note that sets $G_{1}^{1}$ and $G_{1 \mathrm{~L}}^{1} \times G_{1 \mathrm{R}}^{1}$ are trivial in the sense that they only restrict all the elements of a given mass matrix to be real. That is why for categories $L$ and $N$ listed in table 1 the corresponding sets do not include $G_{1}^{1}$ and $G_{1 \mathrm{~L}}^{1} \times G_{1 \mathrm{R}}^{1}$. In these two cases the heavy Majorana neutrino mass matrix $M_{\mathrm{R}}$ has a democracy texture of rank one, and thus its determinant is zero, making the seesaw formula in eq. (2.6) does not work anymore.

It is obvious that if a set contains element $S^{(123)}$, then $M_{\mathrm{D}}, M_{\mathrm{R}}$ and $M_{\nu}$ will all be real. Eq. (2.5) tells us that the structure of $M_{\mathrm{D}}$ is constrained by both $\mathcal{S}_{\mathrm{L}}$ and $\mathcal{S}_{\mathrm{R}}$, and that of $M_{\mathrm{R}}$ is constrained only by $\mathcal{S}_{\mathrm{R}}$. As a result, $M_{\mathrm{D}}$ is constrained more strictly in the case associated with $G_{\mathrm{L}}^{n} \times G_{\mathrm{R}}^{n}$ than in the case associated with $G^{n}$, but the constraints on $M_{\mathrm{R}}$ in these two situations are the same. In fact, $G^{1}$ and $G_{\mathrm{L}}^{1} \times G_{\mathrm{R}}^{1}$ are identical and thus lead to the same textures for relevant mass matrices.

Of course, the structure of $M_{\nu}$ is in general different from that of $M_{\mathrm{R}}$. But as shown in table $1, M_{\nu}$ and $M_{\mathrm{R}}$ do share the same texture for categories $A_{i}$ to $F$, in which the structures of relevant mass matrices are dominated by sets $G^{n}$ and $G_{\mathrm{L}}^{1} \times G_{\mathrm{R}}^{1}$. As for

\footnotetext{
${ }^{2}$ Without invoking any confusion, we have omitted the subscript $i_{1} i_{2} \cdots i_{n}$ (or $\overline{i_{1} i_{2} \cdots i_{6-n}}$ ) of set $G_{i_{1} i_{2} \cdots i_{n}}^{n}$ (or $G_{i_{1} i_{2} \cdots i_{6-n}}^{n}$ ) here and hereafter for the sake of simplicity.
} 
categories $H_{i}$ to $K$, in which sets $G_{\mathrm{L}}^{n} \times G_{\mathrm{R}}^{n}$ (for $n=2,3, \cdots, 6$ ) dominate, the structure of $M_{\nu}$ is quite similar to that of $M_{\mathrm{R}}$ or to a combination of the structures of $M_{\mathrm{D}}$ and $M_{\mathrm{R}}$. In these cases $M_{\mathrm{D}}$ is constrained more strictly than $M_{\mathrm{R}}$, and hence it possesses a much simpler texture which dominates the texture pattern of $M_{\nu}$ via the seesaw formula in eq. (2.6). Especially in categories $I_{i}$ to $K$, the mass matrices $M_{\nu}$ and $M_{\mathrm{D}}$ exactly share the same democracy texture. To characterize the relationship between light and heavy Majorana neutrinos in the seesaw framework under consideration, we refer to the structural equality or similarity between $M_{\nu}$ and $M_{\mathrm{R}}$ as a seesaw mirroring relationship.

Another thing that deserves attention is that the mass matrices constrained by any one of $S^{(123)}, S^{(231)}$ and $S^{(312)}$ must be real. In other words, $M_{\mathrm{D}}$ and $M_{\mathrm{R}}$ will be real if the corresponding set in a given category contains one of the above three elements. Only categories $A_{1}, A_{2}, A_{3}$ and $C$, in which $S^{(123)}, S^{(231)}$ and $S^{(312)}$ are not involved, give rise to complex $M_{\mathrm{D}}$ and $M_{\mathrm{R}}$. Among them, only categories $A_{1}, A_{2}$ and $A_{3}$ allow us to obtain the complex textures of $M_{\nu}$ via the seesaw formula. This observation means that in the $S_{3}$ reflection symmetry limit there are only four possibilities to accommodate CP violation in the lepton-number-violating decays of heavy Majorana neutrinos, and only three possibilities to accommodate CP violation in the effective light neutrino mass matrix $M_{\nu}$.

At this point it is also worth mentioning that $S_{3}$ is not a symmetry of the Lagrangian in the neutrino sector. Although we have considered the subgroups and subsets of $S_{3}$ group, they are mainly used as a tool to constrain and classify possible structures of the neutrino mass matrices. From the phenomenological point of view, our strategy is expected to be helpful for understanding the neutrino flavor structures under $S_{3}$ symmetry and providing a reference about which larger group should be introduced and which representations should be determined when doing a realistic model-building exercise. We admit that a larger flavor symmetry group may not have a direct connection with $S_{3}$, but the latter is likely to play an indirect but suggestive role in bridging an underlying flavor symmetry and a phenomenologically favored pattern of $M_{\nu}$. Since $S_{3}$ is so simple and instructive in reflecting the possible interchange among three flavor families, it should be qualified as a good bottom-up example in probing what is behind tiny neutrino masses and significant flavor mixing effects.

\section{$3 \quad$ Neutrino masses and flavor mixing patterns}

Now we proceed to calculate the light neutrino masses and flavor mixing parameters for each of the textures of $M_{\nu}$ listed in table 1 in the basis where the flavor eigenstates of three charged leptons are identical with their mass eigenstates. Although some of the flavor mixing patterns derived from $M_{\nu}$ in the $S_{3}$ reflection symmetry limit are expected to be far away from the observed pattern of the PMNS matrix, it remains instructive to see their salient features from a phenomenological point of view.

Since $M_{\nu}$ is symmetric, it can be diagonalized by a unitary transformation matrix $V$ as follows: $V^{\dagger} M_{\nu} V^{*}=\widehat{M_{\nu}}$, where $\widehat{M_{\nu}} \equiv \operatorname{Diag}\left\{m_{1}, m_{2}, m_{3}\right\}$ with $m_{i}$ (for $i=1,2,3$ ) being the neutrino masses. In the chosen flavor basis $V$ is just the PMNS matrix which describes 
the effects of neutrino mixing and $\mathrm{CP}$ violation, and its standard parametrization form is

$$
V=P_{l}\left(\begin{array}{ccc}
c_{12} c_{13} & s_{12} c_{13} & s_{13} e^{-\mathrm{i} \delta} \\
-s_{12} c_{23}-c_{12} s_{13} s_{23} e^{\mathrm{i} \delta} & c_{12} c_{23}-s_{12} s_{13} s_{23} e^{\mathrm{i} \delta} & c_{13} s_{23} \\
s_{12} s_{23}-c_{12} s_{13} c_{23} e^{\mathrm{i} \delta} & -c_{12} s_{23}-s_{12} s_{13} c_{23} e^{\mathrm{i} \delta} & c_{13} c_{23}
\end{array}\right) P_{\nu}
$$

where $c_{i j}=\cos \theta_{i j}$ and $s_{i j}=\sin \theta_{i j}$ with $i j=12,13,23, P_{l}=\operatorname{Diag}\left\{e^{\mathrm{i} \phi_{e}}, e^{\mathrm{i} \phi_{\mu}}, e^{\mathrm{i} \phi_{\tau}}\right\}$ contains three unphysical phases which can be absorbed by rephasing the charged-lepton fields, and $P_{\nu}=\operatorname{Diag}\left\{e^{\mathrm{i} \rho}, e^{\mathrm{i} \sigma}, 1\right\}$ contains two physical Majorana phases. Therefore, a diagonalization of the effective Majorana neutrino mass matrix $M_{\nu}$ allows us to determine three neutrino masses $m_{i}$, three flavor mixing angles $\theta_{i j}$ and three CP-violating phases $\delta, \rho$ and $\sigma$. In the following we do such exercises by examining all the textures of $M_{\nu}$ listed in table 1.

\subsection{Categories $A_{i}$}

In category $A_{1}$ the light neutrino mass matrix $M_{\nu}$ satisfies the $\mu-\tau$ reflection symmetry, which naturally predicts the phenomenologically favored results $\theta_{23}=\pi / 4$ and $\delta=$ $-\pi / 2[13,15]$. It is therefore interesting to reproduce this texture from the canonical seesaw mechanism in the $S_{3}$ reflection symmetry limit. To be specific, the $\mu-\tau$ reflection symmetry structure of $M_{\nu}$ in this case leads us to

$$
\begin{array}{rlrl}
\theta_{23}^{A_{1}} & =\frac{\pi}{4}, \quad \delta^{A_{1}} & = \pm \frac{\pi}{2}, \quad \rho^{A_{1}}, \sigma^{A_{1}}=0 \text { or } \frac{\pi}{2}, \\
\phi_{e}^{A_{1}} & =0 \text { or } \frac{\pi}{2}, \quad \phi_{\mu}^{A_{1}}+\phi_{\tau}^{A_{1}}=2 \phi_{e}^{A_{1}} \pm \pi .
\end{array}
$$

In addition, the other two flavor mixing angles and the three neutrino masses in category $A_{1}$ can be expressed as follows:

$$
\begin{aligned}
& \tan \theta_{13}^{A_{1}}=\left|\frac{\operatorname{Im}\left(e^{\prime \prime}\right)}{\sqrt{2} \operatorname{Re}\left(b^{\prime \prime}\right)}\right|, \\
& \tan 2 \theta_{12}^{A_{1}}=\frac{2 \sqrt{2} \cos 2 \theta_{13}^{A_{1}} \operatorname{Re}\left(b^{\prime \prime}\right)}{c_{13}^{A_{1}}\left\{\left[\operatorname{Re}\left(e^{\prime \prime}\right)-d^{\prime \prime}\right] \cos 2 \theta_{13}^{A_{1}}-\left[\operatorname{Re}\left(e^{\prime \prime}\right)+d^{\prime \prime}\right] s_{13}^{A_{1} 2}-a^{\prime \prime} c_{13}^{A_{1} 2}\right\}}, \\
& m_{1}=\left|-d^{\prime \prime}-\frac{\sqrt{2} \operatorname{Re}\left(b^{\prime \prime}\right)}{c_{13}^{A_{1}} \sin 2 \theta_{12}^{A_{1}}}+\frac{\left[a^{\prime \prime}+\operatorname{Re}\left(e^{\prime \prime}\right)+d^{\prime \prime}\right] c_{13}^{A_{1}^{2}}}{2 \cos 2 \theta_{13}^{A_{1}}}\right| \text {, } \\
& m_{2}=\left|-d^{\prime \prime}+\frac{\sqrt{2} \operatorname{Re}\left(b^{\prime \prime}\right)}{c_{13}^{A_{1}} \sin 2 \theta_{12}^{A_{1}}}+\frac{\left[a^{\prime \prime}+\operatorname{Re}\left(e^{\prime \prime}\right)+d^{\prime \prime}\right] c_{13}^{A_{1} 2}}{2 \cos 2 \theta_{13}^{A_{1}}}\right|, \\
& m_{3}=\frac{a^{\prime \prime} s_{13}^{A_{1} 2}+\left[\operatorname{Re}\left(e^{\prime \prime}\right)+d^{\prime \prime}\right] c_{13}^{A_{1} 2}}{\cos 2 \theta_{13}^{A_{1}}},
\end{aligned}
$$

where $a^{\prime \prime}=a_{\mathrm{r}}^{\prime} \exp \left(-2 \mathrm{i} \phi_{e}^{A_{1}}\right), b^{\prime \prime}=b^{\prime} \exp \left[-\mathrm{i}\left(\phi_{e}^{A_{1}}+\phi_{\mu}^{A_{1}}\right)\right], e^{\prime \prime}=e^{\prime} \exp \left(-2 \mathrm{i} \phi_{\mu}^{A_{1}}\right)$ and $d^{\prime \prime}=d_{\mathrm{r}}^{\prime} \exp \left[-\mathrm{i}\left(\phi_{\mu}^{A_{1}}+\phi_{\tau}^{A_{1}}\right)\right]$.

For categories $A_{2}$ and $A_{3}$, the corresponding textures of $M_{\nu}$ are related to that in category $A_{1}$ via eq. (2.16). One may therefore choose the same order of three mass eigenvalues 
and then establish similar correlations among the three PMNS matrices of categories $A_{1}$, $A_{2}$ and $A_{3}$ with the help of eq. (2.16):

$$
V^{A_{2}}=S^{(231)} V^{A_{1}}, \quad V^{A_{3}}=S^{(312)} V^{A_{1}} .
$$

As a consequence, the relevant flavor mixing parameters in categories $A_{2}$ and $A_{3}$ can be respectively related to those of category $A_{1}$ as follows:

$$
\begin{aligned}
& \tan \theta_{23}^{A_{2}}=\frac{1}{\sqrt{2} \tan \theta_{13}^{A_{1}}}, \\
& \sin \theta_{13}^{A_{2}}=\frac{1}{\sqrt{2}} \cos \theta_{13}^{A_{1}} \\
& \cos 2 \theta_{12}^{A_{2}}=-\frac{1-\sin ^{2} \theta_{13}^{A_{1}}}{1+\sin ^{2} \theta_{13}^{A_{1}}} \cos 2 \theta_{12}^{A_{1}}, \\
& \sin \delta^{A_{2}}=\frac{\cos \theta_{12}^{A_{1}} \sin \theta_{12}^{A_{1}} \cos ^{2} \theta_{13}^{A_{1}} \sin \theta_{13}^{A_{1}}}{2 \cos \theta_{12}^{A_{2}} \sin \theta_{12}^{A_{2}} \cos ^{2} \theta_{13}^{A_{2}} \sin \theta_{13}^{A_{2}} \cos \theta_{23}^{A_{2}} \sin \theta_{23}^{A_{2}}} \sin \delta^{A_{1}}, \\
& \phi_{e}^{A_{2}}=\phi_{\mu}^{A_{1}}+\delta^{A_{2}}, \phi_{\mu}^{A_{2}}=\phi_{\tau}^{A_{1}}, \phi_{\tau}^{A_{2}}=\phi_{e}^{A_{1}}-\delta^{A_{1}}, \\
& \rho^{A_{2}}=\varphi_{1}+\rho^{A_{1}}-\delta^{A_{2}}, \sigma^{A_{2}}=\varphi_{2}+\sigma^{A_{1}}-\delta^{A_{2}} ;
\end{aligned}
$$

and

$$
\begin{aligned}
\tan \theta_{23}^{A_{3}} & =\sqrt{2} \tan \theta_{13}^{A_{1}}, \\
\sin \theta_{13}^{A_{3}} & =\frac{1}{\sqrt{2}} \cos \theta_{13}^{A_{1}}, \\
\cos 2 \theta_{12}^{A_{3}} & =-\frac{1-\sin ^{2} \theta_{13}^{A_{1}}}{1+\sin ^{2} \theta_{13}^{A_{1}}} \cos 2 \theta_{12}^{A_{1}}, \\
\sin \delta^{A_{3}} & =\frac{\cos \theta_{12}^{A_{1}} \sin \theta_{12}^{A_{1}} \cos ^{2} \theta_{13}^{A_{1}} \sin \theta_{13}^{A_{1}}}{2 \cos \theta_{12}^{A_{3}} \sin \theta_{12}^{A_{3}} \cos ^{2} \theta_{13}^{A_{3}} \sin \theta_{13}^{A_{3}} \cos \theta_{23}^{A_{3}} \sin \theta_{23}^{A_{3}}} \sin \delta^{A_{1}}, \\
\phi_{e}^{A_{3}} & =\phi_{\tau}^{A_{1}}+\delta^{A_{3}}, \phi_{\mu}^{A_{3}}=\phi_{e}^{A_{1}}-\delta^{A_{1}}, \phi_{\tau}^{A_{3}}=\phi_{\mu}^{A_{1}}, \\
\rho^{A_{3}} & =\pi-\varphi_{1}+\rho^{A_{1}}-\delta^{A_{3}}, \sigma^{A_{3}}=\pi-\varphi_{2}+\sigma^{A_{1}}-\delta^{A_{3}},
\end{aligned}
$$

where $\quad \sin \varphi_{1}=\quad \mp \cos \theta_{12}^{A_{1}} \sin \theta_{13}^{A_{1}} / \sqrt{1-\cos ^{2} \theta_{12}^{A_{1}} \cos ^{2} \theta_{13}^{A_{1}}}, \quad \cos \varphi_{1}=$ $-\sin \theta_{12}^{A_{1}} / \sqrt{1-\cos ^{2} \theta_{12}^{A_{1}} \cos ^{2} \theta_{13}^{A_{1}}}, \quad \sin \varphi_{2}=\mp \sin \theta_{12}^{A_{1}} \sin \theta_{13}^{A_{1}} / \sqrt{1-\sin ^{2} \theta_{12}^{A_{1}} \cos ^{2} \theta_{13}^{A_{1}}}$ and $\cos \varphi_{2}=\cos \theta_{12}^{A_{1}} / \sqrt{1-\sin ^{2} \theta_{12}^{A_{1}} \cos ^{2} \theta_{13}^{A_{1}}}$ with the “干” signs corresponding to $\delta^{A_{1}}= \pm \pi / 2$. The analytical results of three neutrino masses in these two categories are formally the same as those given in eq. (3.3), but of course the relevant flavor mixing parameters need to be substituted with the ones obtained in eq. (3.5) or (3.6). It is obvious that none of the flavor mixing angles and CP-violating phases in categories $A_{2}$ and $A_{3}$ take special values, and this simply means that the standard parametrization of $V$ is not the best choice for these two cases. One may therefore consider to choose another parametrization of $V$ which can automatically reveal the $S_{3}$ reflection symmetry hidden in $M_{\nu}$ in categories $A_{2}$ and $A_{3}$. 


\subsection{Categories $B_{i}$}

In category $B_{1}$ the structure of $M_{\nu}$ possesses the $\mu-\tau$ permutation symmetry, ${ }^{3}$ which naturally predicts $\theta_{13}=0$ and $\theta_{23}=\pi / 4$ in the standard parametrization of $V$ [44-47]. The whole pattern of $V$ in this case is found to be

$$
V^{B_{1}}=\left(\begin{array}{ccc}
\mp \frac{\left(-a_{\mathrm{r}}^{\prime}+e_{\mathrm{r}}^{\prime}+d_{\mathrm{r}}^{\prime}+\sqrt{\Delta}\right)}{\sqrt{8 b_{\mathrm{r}}^{\prime 2}+\left(-a_{\mathrm{r}}^{\prime}+e_{\mathrm{r}}^{\prime}+d_{\mathrm{r}}^{\prime}+\sqrt{\Delta}\right)^{2}}} \pm \frac{\left(a_{\mathrm{r}}^{\prime}-e_{\mathrm{r}}^{\prime}-d_{\mathrm{r}}^{\prime}+\sqrt{\Delta}\right)}{\sqrt{8\left|b_{\mathrm{r}}^{\prime}\right|}} & 0 \\
\frac{2 b_{\mathrm{r}}^{\prime 2}+\left(a_{\mathrm{r}}^{\prime}-e_{\mathrm{r}}^{\prime}-d_{\mathrm{r}}^{\prime}+\sqrt{\Delta}\right)^{2}}{\sqrt{8 b_{\mathrm{r}}^{\prime 2}+\left(-a_{\mathrm{r}}^{\prime}+e_{\mathrm{r}}^{\prime}+d_{\mathrm{r}}^{\prime}+\sqrt{\Delta}\right)^{2}}} & \frac{2\left|b_{\mathrm{r}}^{\prime}\right|}{\sqrt{8 b_{\mathrm{r}}^{\prime 2}+\left(a_{\mathrm{r}}^{\prime}-e_{\mathrm{r}}^{\prime}-d_{\mathrm{r}}^{\prime}+\sqrt{\Delta}\right)^{2}}} & -\frac{1}{\sqrt{2}} \\
\frac{2\left|b_{\mathrm{r}}^{\prime}\right|}{\sqrt{8 b_{\mathrm{r}}^{\prime 2}+\left(-a_{\mathrm{r}}^{\prime}+e_{\mathrm{r}}^{\prime}+d_{\mathrm{r}}^{\prime}+\sqrt{\Delta}\right)^{2}}} & \frac{1}{\sqrt{8 b_{\mathrm{r}}^{\prime 2}+\left(a_{\mathrm{r}}^{\prime}-e_{\mathrm{r}}^{\prime}-d_{\mathrm{r}}^{\prime}+\sqrt{\Delta}\right)^{2}}} & \sqrt{\sqrt{2}}
\end{array}\right)
$$

with two sign options corresponding to the positive or negative sign of $b_{\mathrm{r}}^{\prime}$. It is easy to see that $\left|V_{\mu i}^{B_{1}}\right|=\left|V_{\tau i}^{B_{1}}\right|$ holds (for $i=1,2,3$ ), a clear reflection of the $\mu$ - $\tau$ permutation symmetry. Note, however, that the structure of $M_{\nu}^{B_{1}}$ shown in table 1 is just a particular example which respects the $\mu-\tau$ permutation symmetry but has no complex elements. A general form of $M_{\nu}$ in the $\mu-\tau$ permutation symmetry limit must have a structure similar to $M_{\nu}^{B_{1}}$, but it should contain some complex elements [13].

Given the texture of $M_{\nu}^{B_{1}}$ in table 1 and the pattern of $V^{B_{1}}$ in eq. (3.7), it is straightforward to calculate the neutrino masses and flavor mixing parameters. We obtain

$$
\begin{aligned}
& m_{1}=\frac{1}{2}\left(a_{\mathrm{r}}^{\prime}+e_{\mathrm{r}}^{\prime}+d_{\mathrm{r}}^{\prime}-\sqrt{\Delta}\right), \\
& m_{2}=\frac{1}{2}\left(a_{\mathrm{r}}^{\prime}+e_{\mathrm{r}}^{\prime}+d_{\mathrm{r}}^{\prime}+\sqrt{\Delta}\right), \\
& m_{3}=e_{\mathrm{r}}^{\prime}-d_{\mathrm{r}}^{\prime}
\end{aligned}
$$

and

$$
\begin{aligned}
& \theta_{23}^{B_{1}}=\frac{\pi}{4}, \quad \theta_{13}^{B_{1}}=0, \quad \theta_{12}^{B_{1}}=\arccos \left(\frac{2 \sqrt{2}\left|b_{\mathrm{r}}^{\prime}\right|}{\sqrt{8 b_{\mathrm{r}}^{\prime 2}+\left(a_{\mathrm{r}}^{\prime}-e_{\mathrm{r}}^{\prime}-d_{\mathrm{r}}^{\prime}+\sqrt{\Delta}\right)^{2}}}\right), \\
& \delta^{B_{1}} \in[0,2 \pi), \quad \rho^{B_{1}}=0, \quad \sigma^{B_{1}}=\pi, \quad \phi_{e}^{B_{1}}=0 \text { or } \pi, \quad \phi_{\mu}^{B_{1}}=\pi, \quad \phi_{\tau}^{B_{1}}=0,
\end{aligned}
$$

where $\Delta=8 b_{\mathrm{r}}^{\prime 2}+\left(-a_{\mathrm{r}}^{\prime}+e_{\mathrm{r}}^{\prime}+d_{\mathrm{r}}^{\prime}\right)^{2}$. Note that $m_{1}, m_{2}$ or $m_{3}$ in eq. (3.8) may be negative, but a minus sign can always be absorbed into three unphysical phases and two Majorana phases.

Analogous to categories $A_{1}, A_{2}$ and $A_{3}$, the neutrino masses and flavor mixing parameters of $B_{1}, B_{2}$ and $B_{3}$ are also correlated with one another via eq. (2.16). In fact,

\footnotetext{
${ }^{3}$ That is, the light Majorana neutrino mass term is invariant under the permutation transformations $\nu_{e} \leftrightarrow \nu_{e}$ and $\nu_{\mu} \leftrightarrow \nu_{\tau}$, and thus the structure of $M_{\nu}$ gets constrained.
} 
the three neutrino masses for both categories $B_{2}$ and $B_{3}$ are the same as those given by eq. (3.8), and the flavor mixing parameters in these two cases are found to be

$$
\begin{aligned}
& \theta_{23}^{B_{2}}=\frac{\pi}{2}, \quad \theta_{13}^{B_{2}}=\frac{\pi}{4}, \quad \theta_{12}^{B_{2}}=\arccos \left(\frac{2 \sqrt{2}\left|b_{\mathrm{r}}^{\prime}\right|}{\sqrt{8 b_{\mathrm{r}}^{\prime 2}+\left(-a_{\mathrm{r}}^{\prime}+e_{\mathrm{r}}^{\prime}+d_{\mathrm{r}}^{\prime}+\sqrt{\Delta}\right)^{2}}}\right) \\
& \rho^{B_{2}}=\sigma^{B_{2}}=\pi-\delta^{B_{2}}=-\phi_{e}^{B_{2}}=-\phi_{\tau}^{B_{2}} \text { or } \pi-\phi_{\tau}^{B_{2}}, \quad \phi_{\mu}^{B_{2}}=0
\end{aligned}
$$

and

$$
\begin{aligned}
& \theta_{23}^{B_{3}}=0, \theta_{13}^{B_{3}}=\frac{\pi}{4}, \quad \theta_{12}^{B_{3}}=\arccos \left(\frac{2 \sqrt{2}\left|b_{\mathrm{r}}^{\prime}\right|}{\sqrt{8 b_{\mathrm{r}}^{2}+\left(-a_{\mathrm{r}}^{\prime}+e_{\mathrm{r}}^{\prime}+d_{\mathrm{r}}^{\prime}+\sqrt{\Delta}\right)^{2}}}\right), \\
& \rho^{B_{3}}=\sigma^{B_{3}}=-\delta^{B_{3}}=-\phi_{e}^{B_{3}}=-\phi_{\mu}^{B_{3}} \text { or }-\pi-\phi_{\mu}^{B_{3}}, \quad \phi_{\tau}^{B_{3}}=\pi,
\end{aligned}
$$

respectively. Needless to say, these two possibilities are strongly disfavored by current neutrino oscillation data [48].

\subsection{Categories $C$ and $D$}

The structures of $M_{\nu}$ in categories $C$ and $D$ are exactly the same, and thus their phenomenological consequences are also the same. In particular, they lead us to the well-known tri-bimaximal neutrino mixing pattern [49-51],

$$
V=\left(\begin{array}{ccc}
-\frac{2}{\sqrt{6}} & \frac{1}{\sqrt{3}} & 0 \\
\frac{1}{\sqrt{6}} & \frac{1}{\sqrt{3}} & \frac{1}{\sqrt{2}} \\
\frac{1}{\sqrt{6}} & \frac{1}{\sqrt{3}} & -\frac{1}{\sqrt{2}}
\end{array}\right) .
$$

To be explicit, the results for neutrino masses and flavor mixing parameters are

$$
m_{1}=a_{\mathrm{r}}^{\prime}-b_{\mathrm{r}}^{\prime}, \quad m_{2}=a_{\mathrm{r}}^{\prime}+2 b_{\mathrm{r}}^{\prime}, \quad m_{3}=a_{\mathrm{r}}^{\prime}-b_{\mathrm{r}}^{\prime},
$$

and

$$
\begin{aligned}
\theta_{23} & =\frac{\pi}{4}, & \theta_{13} & =0, \\
\delta & \in[0,2 \pi), & \theta_{12} & =\arctan \left(\frac{1}{\sqrt{2}}\right), \\
& =\pi, & \sigma & =0, \quad \phi_{e}=0, \quad \phi_{\mu}=0, \quad \phi_{\tau}=\pi .
\end{aligned}
$$

So far many model-building exercises have been done in this connection to introduce small perturbations to $M_{\nu}$ and consequently small corrections to $V$ in eq. (3.12), so as to arrive at a better fit of current experimental data [35-40]. 


\subsection{Categories $E_{i}$}

In category $E_{1}$ the PMNS matrix reads

$$
V^{E_{1}}=\left(\begin{array}{ccc}
\frac{2}{\sqrt{6}} & -\frac{1}{\sqrt{3}} & 0 \\
-\frac{1}{\sqrt{6}} & -\frac{1}{\sqrt{3}} & \frac{\mathrm{i}}{\sqrt{2}} \\
-\frac{1}{\sqrt{6}} & -\frac{1}{\sqrt{3}} & -\frac{\mathrm{i}}{\sqrt{2}}
\end{array}\right)
$$

which is also the tri-bimaximal flavor mixing pattern with some trivial phases. A straightforward calculation allows us to obtain the neutrino masses for category $E_{1}$ :

$$
m_{1}=a_{\mathrm{r}}^{\prime}-b_{\mathrm{r}}^{\prime}, \quad m_{2}=a_{\mathrm{r}}^{\prime}+2 b_{\mathrm{r}}^{\prime}, \quad m_{3}=a_{\mathrm{r}}^{\prime}-b_{\mathrm{r}}^{\prime},
$$

and the same result is true for categories $E_{2}$ and $E_{3}$. The corresponding flavor mixing parameters are found to be

$$
\begin{array}{rlrl}
\theta_{23}^{E_{1}} & =\frac{\pi}{4}, & \theta_{13}^{E_{1}} & =0, \\
\delta^{E_{1}} & \in[0,2 \pi), & \rho^{E_{1}}=\frac{3 \pi}{2}, & \theta_{12}^{E_{1}}=\arctan \left(\frac{1}{\sqrt{2}}\right), \\
\phi_{e}^{E_{1}}=\frac{\pi}{2}, & \phi_{\mu}^{E_{1}}=\frac{\pi}{2}, & \phi_{\tau}^{E_{1}}=\frac{\pi}{2},
\end{array}
$$

in category $E_{1}$;

$$
\begin{aligned}
& \theta_{23}^{E_{2}}=\frac{\pi}{2}, \quad \theta_{13}^{E_{2}}=\frac{\pi}{4}, \quad \theta_{12}^{E_{2}}=\arctan (\sqrt{2}) \\
& \rho^{E_{2}}=\sigma^{E_{2}}=\frac{\pi}{2}-\delta^{E_{2}}=\pi-\phi_{e}^{E_{2}}=-\phi_{\tau}^{E_{2}}, \quad \phi_{\mu}^{E_{2}}=\frac{3 \pi}{2}
\end{aligned}
$$

in category $E_{2}$; and

$$
\begin{aligned}
& \theta_{23}^{E_{3}}=0, \quad \theta_{13}^{E_{3}}=\frac{\pi}{4}, \quad \theta_{12}^{E_{3}}=\arctan (\sqrt{2}), \\
& \rho^{E_{3}}=\sigma^{E_{3}}=\frac{3 \pi}{2}-\delta^{E_{3}}=\pi-\phi_{e}^{E_{3}}=\pi-\phi_{\mu}^{E_{3}}, \quad \phi_{\tau}^{E_{3}}=\frac{\pi}{2}
\end{aligned}
$$

in category $E_{3}$. One can see that the latter two cases are strongly disfavored by current neutrino oscillation data [48].

\subsection{Category $F$}

In this category of $M_{\nu}$ the PMNS matrix is given by

$$
V=\left(\begin{array}{ccc}
\mathrm{i} \frac{-a_{\mathrm{r}}^{\prime}+b_{\mathrm{r}}^{\prime}+\lambda}{a_{\mathrm{r}}^{\prime}-e_{\mathrm{r}}^{\prime}} \sqrt{\frac{-2 b_{\mathrm{r}}^{\prime}+a_{\mathrm{r}}^{\prime}+e_{\mathrm{r}}^{\prime}+2 \lambda}{6 \lambda}} & \frac{-a_{\mathrm{r}}^{\prime}+b_{\mathrm{r}}^{\prime}-\lambda}{a_{\mathrm{r}}^{\prime}-e_{\mathrm{r}}^{\prime}} \sqrt{\frac{2 b_{\mathrm{r}}^{\prime}-a_{\mathrm{r}}^{\prime}-e_{\mathrm{r}}^{\prime}+2 \lambda}{6 \lambda}} & \frac{1}{\sqrt{3}} \\
\mathrm{i} \frac{-b_{\mathrm{r}}^{\prime}+e_{\mathrm{r}}^{\prime}-\lambda}{a_{\mathrm{r}}^{\prime}-e_{\mathrm{r}}^{\prime}} \sqrt{\frac{-2 b_{\mathrm{r}}^{\prime}+a_{\mathrm{r}}^{\prime}+e_{\mathrm{r}}^{\prime}+2 \lambda}{6 \lambda}} & \frac{-b_{\mathrm{r}}^{\prime}+e_{\mathrm{r}}^{\prime}+\lambda}{a_{\mathrm{r}}^{\prime}-e_{\mathrm{r}}^{\prime}} \sqrt{\frac{2 b_{\mathrm{r}}^{\prime}-a_{\mathrm{r}}^{\prime}-e_{\mathrm{r}}^{\prime}+2 \lambda}{6 \lambda}} & \frac{1}{\sqrt{3}} \\
\mathrm{i} \sqrt{\frac{-2 b_{\mathrm{r}}^{\prime}+a_{\mathrm{r}}^{\prime}+e_{\mathrm{r}}^{\prime}+2 \lambda}{6 \lambda}} & \sqrt{\frac{2 b_{\mathrm{r}}^{\prime}-a_{\mathrm{r}}^{\prime}-e_{\mathrm{r}}^{\prime}+2 \lambda}{6 \lambda}} & \frac{1}{\sqrt{3}}
\end{array}\right)
$$


with $\lambda=\sqrt{a_{\mathrm{r}}^{\prime 2}+b_{\mathrm{r}}^{\prime 2}+e_{\mathrm{r}}^{\prime 2}-a_{\mathrm{r}}^{\prime} b_{\mathrm{r}}^{\prime}-a_{\mathrm{r}}^{\prime} e_{\mathrm{r}}^{\prime}-b_{\mathrm{r}}^{\prime} e_{\mathrm{r}}^{\prime}}$. The masses of three light neutrinos are

$$
m_{1}=m_{2}=\lambda, \quad m_{3}=a_{\mathrm{r}}^{\prime}+b_{\mathrm{r}}^{\prime}+e_{\mathrm{r}}^{\prime},
$$

and the corresponding flavor mixing parameters are found to be

$$
\begin{aligned}
\theta_{23} & =\frac{\pi}{4}, \quad \theta_{13}=\arccos \left(\frac{2}{\sqrt{6}}\right), \\
\theta_{12} & =\arctan \left(\frac{a-b+\lambda}{a-b-\lambda} \sqrt{\frac{2 b_{\mathrm{r}}^{\prime}-a_{\mathrm{r}}^{\prime}-e_{\mathrm{r}}^{\prime}+2 \lambda}{-2 b_{\mathrm{r}}^{\prime}+a_{\mathrm{r}}^{\prime}+e_{\mathrm{r}}^{\prime}+2 \lambda}}\right), \\
\delta & =0 \text { or } \pi, \quad \rho=\frac{\pi}{2}-\delta, \quad \sigma=\phi_{e}=\delta, \quad \phi_{\mu}=\phi_{\tau}=0 .
\end{aligned}
$$

This case turns out to be strongly disfavored by current experimental data.

\subsection{Categories $H_{i}$}

For categories $H_{1}, H_{2}$ and $H_{3}$, the corresponding neutrino masses and flavor mixing parameters can easily be obtained from categories $B_{1}, B_{2}$ and $B_{3}$ by taking $d_{\mathrm{r}}^{\prime}=e_{\mathrm{r}}^{\prime}$. In this way one is left with $m_{3}=0$, corresponding to the inverted neutrino mass hierarchy. Here let us focus on the normal neutrino mass hierarchy. The PMNS matrix is found to be

$$
V^{H_{1}}=\left(\begin{array}{ccc}
0 & \mp \frac{\left(-a_{\mathrm{r}}^{\prime}+2 e_{\mathrm{r}}^{\prime}+\sqrt{\Delta^{\prime}}\right)}{\sqrt{8 b_{\mathrm{r}}^{\prime 2}+\left(-a_{\mathrm{r}}^{\prime}+2 e_{\mathrm{r}}^{\prime}+\sqrt{\Delta^{\prime}}\right)^{2}}} \pm \frac{\left(a_{\mathrm{r}}^{\prime}-2 e_{\mathrm{r}}^{\prime}+\sqrt{\Delta^{\prime}}\right)}{\sqrt{8 b_{\mathrm{r}}^{\prime 2}+\left(a_{\mathrm{r}}^{\prime}-2 e_{\mathrm{r}}^{\prime}+\sqrt{\Delta^{\prime}}\right)^{2}}} \\
-\frac{1}{\sqrt{2}} & \frac{2\left|b_{\mathrm{r}}^{\prime}\right|}{\sqrt{8 b_{\mathrm{r}}^{\prime 2}+\left(-a_{\mathrm{r}}^{\prime}+2 e_{\mathrm{r}}^{\prime}+\sqrt{\Delta^{\prime}}\right)^{2}}} & \frac{2\left|b_{\mathrm{r}}^{\prime}\right|}{\sqrt{8 b_{\mathrm{r}}^{\prime 2}+\left(a_{\mathrm{r}}^{\prime}-2 e_{\mathrm{r}}^{\prime}+\sqrt{\Delta^{\prime}}\right)^{2}}} \\
\frac{1}{\sqrt{2}} & \frac{2\left|b_{\mathrm{r}}^{\prime}\right|}{\sqrt{8 b_{\mathrm{r}}^{\prime 2}+\left(-a_{\mathrm{r}}^{\prime}+2 e_{\mathrm{r}}^{\prime}+\sqrt{\Delta^{\prime}}\right)^{2}}} & \frac{1}{\sqrt{8 b_{\mathrm{r}}^{\prime 2}+\left(a_{\mathrm{r}}^{\prime}-2 e_{\mathrm{r}}^{\prime}+\sqrt{\Delta^{\prime}}\right)^{2}}}
\end{array}\right),
$$

and the neutrino masses are given by

$$
\begin{aligned}
& m_{1}=0, \\
& m_{2}=\frac{1}{2}\left(a_{\mathrm{r}}^{\prime}+2 e_{\mathrm{r}}^{\prime}-\sqrt{\Delta^{\prime}}\right), \\
& m_{3}=\frac{1}{2}\left(a_{\mathrm{r}}^{\prime}+2 e_{\mathrm{r}}^{\prime}+\sqrt{\Delta^{\prime}}\right),
\end{aligned}
$$

where $\Delta^{\prime}=8 b_{\mathrm{r}}^{\prime 2}+\left(-a_{\mathrm{r}}^{\prime}+2 e_{\mathrm{r}}^{\prime}\right)^{2}$. The results of $m_{i}$ in eq. (3.24) are also valid for categories $H_{2}$ and $H_{3}$. To be explicit, the flavor mixing parameters in these three cases are obtained below:

$$
\begin{aligned}
& \theta_{23}^{H_{1}}=\frac{\pi}{4}, \quad \theta_{12}^{H_{1}}=\frac{\pi}{2}, \quad \theta_{13}^{H_{1}}=\arccos \left(\frac{2 \sqrt{2}\left|b_{\mathrm{r}}^{\prime}\right|}{\sqrt{8 b_{\mathrm{r}}^{\prime 2}+\left(a_{\mathrm{r}}^{\prime}-2 e_{\mathrm{r}}^{\prime}+\sqrt{\Delta^{\prime}}\right)^{2}}}\right), \\
& \delta^{H_{1}}=\pi-\sigma^{H_{1}}=\phi_{e}^{H_{1}} \text { or } \phi_{e}^{H_{1}}+\pi, \quad \rho^{H_{1}}=\phi_{\mu}^{H_{1}}=\phi_{\tau}^{H_{1}}=0
\end{aligned}
$$


for category $H_{1}$;

$$
\begin{aligned}
& \theta_{23}^{H_{2}}=\arctan \left(\frac{2\left|b_{\mathrm{r}}^{\prime}\right|}{a_{\mathrm{r}}^{\prime}-2 e_{\mathrm{r}}^{\prime}+\sqrt{\Delta^{\prime}}}\right), \\
& \theta_{12}^{H_{2}}=\arctan \left(\frac{2 \sqrt{2}\left|b_{\mathrm{r}}^{\prime}\right|}{\sqrt{8 b_{\mathrm{r}}^{\prime 2}+\left(-a_{\mathrm{r}}^{\prime}+2 e_{\mathrm{r}}^{\prime}+\sqrt{\Delta^{\prime}}\right)^{2}}}\right), \\
& \theta_{13}^{H_{2}}=\arcsin \left(\frac{2\left|b_{\mathrm{r}}^{\prime}\right|}{\sqrt{8 b_{\mathrm{r}}^{\prime 2}+\left(a_{\mathrm{r}}^{\prime}-2 e_{\mathrm{r}}^{\prime}+\sqrt{\Delta^{\prime}}\right)^{2}}}\right), \\
& \delta^{H_{2}}=\sigma^{H_{2}}=\phi_{e}^{H_{2}}=\phi_{\mu}^{H_{2}}=0, \quad \rho^{H_{2}}=\pi, \quad \phi_{\tau}^{H_{2}}=0 \text { or }-\pi
\end{aligned}
$$

for category $\mathrm{H}_{2}$; and

$$
\begin{aligned}
& \theta_{23}^{H_{3}}=\arctan \left(\frac{a_{\mathrm{r}}^{\prime}-2 e_{\mathrm{r}}^{\prime}+\sqrt{\Delta^{\prime}}}{2\left|b_{\mathrm{r}}^{\prime}\right|}\right), \\
& \theta_{12}^{H_{3}}=\arctan \left(\frac{2 \sqrt{2}\left|b_{\mathrm{r}}^{\prime}\right|}{\sqrt{8 b_{\mathrm{r}}^{\prime 2}+\left(-a_{\mathrm{r}}^{\prime}+2 e_{\mathrm{r}}^{\prime}+\sqrt{\Delta^{\prime}}\right)^{2}}}\right), \\
& \theta_{13}^{H_{3}}=\arcsin \left(\frac{2\left|b_{\mathrm{r}}^{\prime}\right|}{\sqrt{8 b_{\mathrm{r}}^{\prime 2}+\left(a_{\mathrm{r}}^{\prime}-2 e_{\mathrm{r}}^{\prime}+\sqrt{\Delta^{\prime}}\right)^{2}}}\right), \\
& \delta^{H_{3}}=\rho^{H_{3}}=\sigma^{H_{3}}=\phi_{e}^{H_{3}}=\pi, \quad \phi_{\tau}^{H_{3}}=0, \quad \phi_{\mu}^{H_{3}}=0 \text { or }-\pi
\end{aligned}
$$

for category $H_{3}$, respectively. Note that the flavor mixing angles in the latter two cases satisfy the relations $\tan \theta_{12}^{H_{2}} \tan \theta_{23}^{H_{2}}=\sin \theta_{13}^{H_{2}}$ and $\tan \theta_{12}^{H_{3}}=\tan \theta_{23}^{H_{3}} \sin \theta_{13}^{H_{3}}$.

\subsection{Categories $I_{i}, J$ and $K$}

In these five categories the textures of $M_{\nu}$ are all democratic, and thus the corresponding PMNS matrix is of the form

$$
V=\left(\begin{array}{ccc}
\frac{1}{\sqrt{2}} & \frac{1}{\sqrt{6}} & \frac{1}{\sqrt{3}} \\
-\frac{1}{\sqrt{2}} & \frac{1}{\sqrt{6}} & \frac{1}{\sqrt{3}} \\
0 & -\frac{2}{\sqrt{6}} & \frac{1}{\sqrt{3}}
\end{array}\right),
$$

corresponding to a special neutrino mass spectrum with $m_{1}=m_{2}=0$ and $m_{3}=3 a_{\mathrm{r}}^{\prime}$. To be explicit, the pattern of $V$ in eq. (3.28) leads us to

$$
\begin{aligned}
\theta_{23} & =\frac{\pi}{4}, \quad \theta_{13}=\arccos \left(\frac{2}{\sqrt{6}}\right), \quad \theta_{12}=\frac{\pi}{6}, \\
\delta & =\rho=\sigma=\phi_{e}=\phi_{\mu}=\phi_{\tau}=0,
\end{aligned}
$$

which are strongly disfavored by current neutrino oscillation data. 


\section{Leptogenesis in the $S_{3}$ symmetry limit}

Now we examine whether the leptogenesis mechanism $[16],{ }^{4}$ which can provide a natural way to account for the observed matter-antimatter asymmetry of the Universe [53], works or not in the $S_{3}$ reflection symmetry limit under discussion. According to this mechanism, the lepton-number-violating, CP-violating and out-of-equilibrium decays of heavy Majorana neutrinos $N_{i}$ may result in a lepton-antilepton asymmetry in the early Universe, and the latter can subsequently be converted to the wanted baryon-antibaryon asymmetry through the $B-L$ conserving sphaleron process $[54,55]$. Here what we are concerned with are the CP-violating asymmetries between the decay modes $N_{i} \rightarrow \ell_{\alpha}+H$ and their CP-conjugate processes $N_{i} \rightarrow \bar{\ell}_{\alpha}+\bar{H}$, usually denoted as $\epsilon_{i \alpha}$ (for $\alpha=e, \mu, \tau$ and $i=1,2,3$ ), because they will finally determine the strength of baryogenesis via leptogenesis. Assuming that the masses of three heavy Majorana neutrinos are hierarchical (i.e., $M_{1} \ll M_{2}<M_{3}$ ), it has been shown that only the CP-violating asymmetries $\epsilon_{1 \alpha}$ survive and contribute to the lepton-antilepton asymmetry. In this case the expression of $\epsilon_{1 \alpha}$ is given by $[42,56]$

$$
\begin{aligned}
\epsilon_{1 \alpha}= & \frac{\Gamma\left(N_{i} \rightarrow \ell_{\alpha}+H\right)-\Gamma\left(N_{i} \rightarrow \bar{\ell}_{\alpha}+\bar{H}\right)}{\sum_{\alpha}\left[\Gamma\left(N_{i} \rightarrow \ell_{\alpha}+H\right)+\Gamma\left(N_{i} \rightarrow \bar{\ell}_{\alpha}+\bar{H}\right)\right]} \\
= & \frac{1}{8 \pi v^{2}\left(\widetilde{M}_{\mathrm{D}}^{\dagger} \widetilde{M}_{\mathrm{D}}\right)_{11}} \sum_{j \neq 1}\left\{\operatorname{Im}\left[\left(\widetilde{M}_{\mathrm{D}}^{*}\right)_{\alpha 1}\left(\widetilde{M}_{\mathrm{D}}\right)_{\alpha j}\left(\widetilde{M}_{\mathrm{D}}^{\dagger} \widetilde{M}_{\mathrm{D}}\right)_{1 j}\right] \times \mathcal{F}\left(\frac{M_{j}^{2}}{M_{1}^{2}}\right)\right. \\
& \left.\quad+\operatorname{Im}\left[\left(\widetilde{M}_{\mathrm{D}}^{*}\right)_{\alpha 1}\left(\widetilde{M}_{\mathrm{D}}\right)_{\alpha j}\left(\widetilde{M}_{\mathrm{D}}^{\dagger} \widetilde{M}_{\mathrm{D}}\right)_{1 j}^{*}\right] \times \mathcal{G}\left(\frac{M_{j}^{2}}{M_{1}^{2}}\right)\right\},
\end{aligned}
$$

where $\widetilde{M}_{\mathrm{D}}=M_{\mathrm{D}} U_{\mathrm{R}}^{*}$ with $U_{\mathrm{R}}$ being the unitary matrix used to diagonalize $M_{\mathrm{R}}$ (i.e., $\left.U_{\mathrm{R}}^{\dagger} M_{\mathrm{R}} U_{\mathrm{R}}^{*}=\widehat{M}_{N}=\operatorname{Diag}\left\{M_{1}, M_{2}, M_{3}\right\}\right), M_{i}$ (for $i=1,2,3$ ) stands for the mass of the heavy Majorana neutrino $N_{i}, v \equiv\langle H\rangle \approx 174 \mathrm{GeV}$ is the vacuum expectation value of the Higgs field, $\mathcal{F}(x)$ and $\mathcal{G}(x)$ are the loop functions defined as $\mathcal{F}(x)=$ $\sqrt{x}\{(2-x) /(1-x)+(1+x) \ln [x /(1+x)]\}$ and $\mathcal{G}(x)=1 /(1-x)$, respectively. If all the interactions in the period of leptogenesis are blind to lepton flavors, then only the total CP-violating asymmetry $\epsilon_{1}$ is relevant,

$$
\epsilon_{1}=\sum_{\alpha} \epsilon_{1 \alpha}=\frac{1}{8 \pi v^{2}\left(\widetilde{M}_{\mathrm{D}}^{\dagger} \widetilde{M}_{\mathrm{D}}\right)_{11}} \sum_{j \neq 1} \operatorname{Im}\left[\left(\widetilde{M}_{\mathrm{D}}^{\dagger} \widetilde{M}_{\mathrm{D}}\right)_{1 j}^{2}\right] \times \mathcal{F}\left(\frac{M_{j}^{2}}{M_{1}^{2}}\right)
$$

In the literature $\epsilon_{1}$ and $\epsilon_{1 \alpha}$ correspond to the so-called "unflavored" and "flavored" leptogenesis. In view of table 1 , it is obvious that only categories $A_{i}$ (for $i=1,2,3$ ) and $C$ are likely to lead us to nonzero $\epsilon_{1}$ or $\epsilon_{1 \alpha}$, and thus we are going to calculate them in the following.

\footnotetext{
${ }^{4}$ For a review, see e.g. ref. [52].
} 


\subsection{Unflavored leptogenesis}

\subsubsection{Categories $A_{i}$}

Let us first consider category $A_{1}$, and then turn to categories $A_{2}$ and $A_{3}$. In category $A_{1}$ the three mass matrices all respect the $\mu-\tau$ reflection symmetry, so it is easy to calculate their corresponding mass eigenvalues and flavor mixing parameters. To be more specific, the unitary matrix $U_{\mathrm{R}}$ used to diagonalize $M_{\mathrm{R}}$ can be decomposed as $U_{\mathrm{R}}=P_{1}^{\mathrm{R}} \widetilde{U}^{\mathrm{R}} P_{2}^{\mathrm{R}}$, where $\widetilde{U}^{\mathrm{R}}=O_{23} \tilde{O}_{13} O_{12}$ is of the same form as the standard parameterization shown in eq. (3.1), and $P_{1}^{\mathrm{R}}=\operatorname{Diag}\left\{e^{\mathrm{i} \phi_{1}^{\mathrm{R}}}, e^{\mathrm{i} \phi_{2}^{\mathrm{R}}}, e^{\mathrm{i} \phi_{3}^{\mathrm{R}}}\right\}$ and $P_{2}^{\mathrm{R}}=\operatorname{Diag}\left\{e^{\mathrm{i} \rho^{\mathrm{R}}}, e^{\mathrm{i} \sigma^{\mathrm{R}}}, 1\right\}$ are the diagonal phase matrices. Then we obtain

$$
\begin{aligned}
& \theta_{23}^{\mathrm{R}}=\frac{\pi}{4} \quad \delta^{\mathrm{R}}= \pm \frac{\pi}{2}, \quad \rho^{\mathrm{R}}, \sigma^{\mathrm{R}}=0 \text { or } \frac{\pi}{2}, \\
& \phi_{1}^{\mathrm{R}}=0 \text { or } \frac{\pi}{2}, \quad \phi_{2}^{\mathrm{R}}+\phi_{3}^{\mathrm{R}}=2 \phi_{1}^{\mathrm{R}} \pm \pi .
\end{aligned}
$$

One can see that all the phase parameters take very special values.

We proceed to calculate the elements $\left(\widetilde{M}_{\mathrm{D}}^{\dagger} \widetilde{M}_{\mathrm{D}}\right)_{1 j}$ which appear in eq. (4.2). The Hermitian matrix $\widetilde{M}_{\mathrm{D}}^{\dagger} \widetilde{M}_{\mathrm{D}}$ can be rewritten as

$$
\widetilde{M}_{\mathrm{D}}^{\dagger} \widetilde{M}_{\mathrm{D}}=U_{\mathrm{R}}^{\mathrm{T}} M_{\mathrm{D}}^{\dagger} M_{\mathrm{D}} U_{\mathrm{R}}^{*}=U_{\mathrm{R}}^{\mathrm{T}} U_{23} U_{23}^{\dagger} M_{\mathrm{D}}^{\dagger} M_{\mathrm{D}} U_{23} U_{23}^{\dagger} U_{\mathrm{R}}^{*}=U_{\mathrm{R}}^{\prime \mathrm{T}} H U_{\mathrm{R}}^{\prime *},
$$

in which

$$
U_{23}=\left(\begin{array}{ccc}
1 & 0 & 0 \\
0 & \frac{\mathrm{i}}{\sqrt{2}} & \frac{1}{\sqrt{2}} \\
0 & \frac{-\mathrm{i}}{\sqrt{2}} & \frac{1}{\sqrt{2}}
\end{array}\right),
$$

and

$$
H=U_{23}^{\dagger} M_{\mathrm{D}}^{\dagger} M_{\mathrm{D}} U_{23}=\left(\begin{array}{lll}
A^{\prime} & B^{\prime} & C^{\prime} \\
B^{\prime} & E^{\prime} & D^{\prime} \\
C^{\prime} & D^{\prime} & F^{\prime}
\end{array}\right)
$$

is a real symmetric matrix whose elements are given by

$$
\begin{aligned}
& A^{\prime}=A_{\mathrm{r}}^{2}+2|E|^{2}, \\
& B^{\prime}=-\sqrt{2} \operatorname{Im}\left(A_{\mathrm{r}} B+E^{*} C+E D^{*}\right), \\
& C^{\prime}=\sqrt{2} \operatorname{Re}\left(A_{\mathrm{r}} B+E^{*} C+E D^{*}\right), \\
& D^{\prime}=-\operatorname{Im}\left(B^{2}+2 C D^{*}\right), \\
& E^{\prime}=|B|^{2}+|C|^{2}+|D|^{2}-\operatorname{Re}\left(B^{2}+2 C D^{*}\right), \\
& F^{\prime}=|B|^{2}+|C|^{2}+|D|^{2}+\operatorname{Re}\left(B^{2}+2 C D^{*}\right),
\end{aligned}
$$

and finally

$$
U_{\mathrm{R}}^{\prime}=U_{23}^{T} U_{\mathrm{R}}=\left(\begin{array}{ccc}
\eta & 0 & 0 \\
0 & \mathrm{i} x & \mathrm{i} y \\
0 & y & x
\end{array}\right)\left(\begin{array}{ccc}
c_{13}^{\mathrm{R}} c_{12}^{\mathrm{R}} & c_{13}^{\mathrm{R}} s_{12}^{\mathrm{R}} & s_{13}^{\mathrm{R}} e^{-\mathrm{i} \delta^{\mathrm{R}}} \\
-s_{12}^{\mathrm{R}} & c_{12}^{\mathrm{R}} & 0 \\
-s_{13}^{\mathrm{R}} c_{12}^{\mathrm{R}} e^{\mathrm{i} \delta^{\mathrm{R}}} & -s_{13}^{\mathrm{R}} s_{12}^{\mathrm{R}} e^{\mathrm{i} \delta^{\mathrm{R}}} & c_{13}^{\mathrm{R}}
\end{array}\right)\left(\begin{array}{ccc}
e^{\mathrm{i} \rho^{\mathrm{R}}} & 0 & 0 \\
0 & e^{\mathrm{i} \sigma^{\mathrm{R}}} & 0 \\
0 & 0 & 1
\end{array}\right)
$$


with $c_{i j}^{\mathrm{R}}=\cos \theta_{i j}^{\mathrm{R}}, s_{i j}^{\mathrm{R}}=\sin \theta_{i j}^{\mathrm{R}}$, and $x=\mathrm{i} \sin \phi_{2}^{\mathrm{R}}$ and $y=\cos \phi_{2}^{\mathrm{R}}$ for $\eta=1$ (i.e., $\phi_{1}^{\mathrm{R}}=0$ ) or $x=\cos \phi_{2}^{\mathrm{R}}$ and $y=\mathrm{i} \sin \phi_{2}^{\mathrm{R}}$ for $\eta=\mathrm{i}$ (i.e., $\phi_{1}^{\mathrm{R}}=\pi / 2$ ). With the help of eqs. (4.6) and (4.8), eq. (4.4) can be expressed as

$$
\left(\widetilde{M}_{\mathrm{D}}^{\dagger} \widetilde{M}_{\mathrm{D}}\right)_{1 j}=\sum_{k=1}^{3} W_{k}\left(\begin{array}{ccc}
c_{13}^{\mathrm{R}} c_{12}^{\mathrm{R}} & c_{13}^{\mathrm{R}} s_{12}^{\mathrm{R}} & s_{13}^{\mathrm{R}} e^{\mathrm{i} \delta^{\mathrm{R}}} \\
-s_{12}^{\mathrm{R}} & c_{12}^{\mathrm{R}} & 0 \\
-s_{13}^{\mathrm{R}} c_{12}^{\mathrm{R}} e^{-\mathrm{i} \delta^{\mathrm{R}}} & -s_{13}^{\mathrm{R}} s_{12}^{\mathrm{R}} e^{-\mathrm{i} \delta^{\mathrm{R}}} & c_{13}^{\mathrm{R}}
\end{array}\right)_{k j} P_{j j},
$$

in which $P=\operatorname{Diag}\left\{1, e^{\mathrm{i}\left(\rho^{\mathrm{R}}-\sigma^{\mathrm{R}}\right)}, e^{\mathrm{i} \rho^{\mathrm{R}}}\right\}$ and

$$
W_{k}=\left[\left(\begin{array}{ccc}
c_{13}^{\mathrm{R}} c_{12}^{\mathrm{R}} & -s_{12}^{\mathrm{R}} & -s_{13}^{\mathrm{R}} c_{12}^{\mathrm{R}} e^{\mathrm{i} \delta^{\mathrm{R}}} \\
c_{13}^{\mathrm{R}} s_{12}^{\mathrm{R}} & c_{12}^{\mathrm{R}} & -s_{13}^{\mathrm{R}} s_{12}^{\mathrm{R}} e^{\mathrm{i} \delta^{\mathrm{R}}} \\
s_{13}^{\mathrm{R}} e^{-\mathrm{i} \delta^{\mathrm{R}}} & 0 & c_{13}^{\mathrm{R}}
\end{array}\right)\left(\begin{array}{ccc}
\eta & 0 & 0 \\
0 & \mathrm{i} x & y \\
0 & \mathrm{i} y & x
\end{array}\right) H\left(\begin{array}{ccc}
\eta^{*} & 0 & 0 \\
0 & -\mathrm{i} x^{*} & -\mathrm{i} y^{*} \\
0 & y^{*} & x^{*}
\end{array}\right)\right]_{1 k}
$$

Concretely,

$$
\begin{aligned}
& W_{1}=A^{\prime} c_{12}^{\mathrm{R}} c_{13}^{\mathrm{R}}-p s_{12}-q s_{13}^{\mathrm{R}} c_{12}^{\mathrm{R}} e^{\mathrm{i} \delta^{\mathrm{R}}}, \\
& W_{2}=p^{*} c_{12}^{\mathrm{R}} c_{13}^{\mathrm{R}}-t_{1} s_{12}-r s_{13}^{\mathrm{R}} c_{12}^{\mathrm{R}} e^{\mathrm{i} \delta^{\mathrm{R}}}, \\
& W_{3}=q^{*} c_{12}^{\mathrm{R}} c_{13}^{\mathrm{R}}-r^{*} s_{12}-t_{2} s_{13}^{\mathrm{R}} c_{12}^{\mathrm{R}} e^{\mathrm{i} \delta^{\mathrm{R}}},
\end{aligned}
$$

where

$$
\begin{aligned}
p & =\eta^{*}\left(\mathrm{i} x B^{\prime}+y C^{\prime}\right), \\
q & =\eta^{*}\left(\mathrm{i} y B^{\prime}+x C^{\prime}\right), \\
t_{1} & =|x|^{2} E^{\prime}+|y|^{2} F^{\prime}-2 D^{\prime} \operatorname{Im}\left(x y^{*}\right), \\
t_{2} & =|x|^{2} F^{\prime}+|y|^{2} E^{\prime}+2 D^{\prime} \operatorname{Im}\left(x y^{*}\right), \\
r & =x^{*} y E^{\prime}+x y^{*} F^{\prime}-\mathrm{i} D^{\prime}\left(|x|^{2}-|y|^{2}\right),
\end{aligned}
$$

with $t_{1}$ and $t_{2}$ being real. Taking account of eqs. (4.9)-(4.12), we obtain

$$
\begin{aligned}
& \left(\widetilde{M}_{\mathrm{D}}^{\dagger} \widetilde{M}_{\mathrm{D}}\right)_{11}=A^{\prime 2} c_{12}^{\mathrm{R} 2} c_{13}^{\mathrm{R} 2}-2 s_{12}^{\mathrm{R}} c_{12}^{\mathrm{R}} c_{13}^{\mathrm{R}} \operatorname{Re}(p)-2 \mathrm{i} e^{\mathrm{i} \delta^{R}} s_{13}^{\mathrm{R}} c_{13}^{\mathrm{R}} c_{12}^{\mathrm{R} 2} \operatorname{Im}(q) \\
& +2 \mathrm{i} e^{\mathrm{i} \delta^{R}} s_{13}^{\mathrm{R}} s_{12}^{\mathrm{R}} c_{12}^{\mathrm{R}} \operatorname{Im}(r)+t_{1} s_{12}^{\mathrm{R} 2}+t_{2} s_{13}^{\mathrm{R} 2} c_{12}^{\mathrm{R} 2}, \\
& \left(\widetilde{M}_{\mathrm{D}}^{\dagger} \widetilde{M}_{\mathrm{D}}\right)_{12}=e^{\mathrm{i}\left(\rho^{\mathrm{R}}-\sigma^{\mathrm{R}}\right)}\left[A^{\prime} s_{12}^{\mathrm{R}} c_{12}^{\mathrm{R}} c_{13}^{\mathrm{R} 2}-2 s_{12}^{\mathrm{R} 2} c_{13}^{\mathrm{R}} \operatorname{Re}(p)+p^{*} c_{13}^{\mathrm{R}}\right. \\
& -2 \mathrm{i} e^{\mathrm{i} \delta^{R}} s_{13}^{\mathrm{R}} c_{13}^{\mathrm{R}} s_{12}^{\mathrm{R}} c_{12}^{\mathrm{R}} \operatorname{Im}(q)+2 \mathrm{i} e^{\mathrm{i} \delta^{R}} s_{13}^{\mathrm{R}} s_{12}^{\mathrm{R} 2} \operatorname{Im}(r) \\
& \left.-r e^{\mathrm{i} \delta^{R}} s_{13}^{\mathrm{R}}-t_{1} s_{12}^{\mathrm{R}} c_{12}^{\mathrm{R}}+t_{2} s_{13}^{\mathrm{R} 2} s_{12}^{\mathrm{R}} c_{12}^{\mathrm{R}}\right], \\
& \left(\widetilde{M}_{\mathrm{D}}^{\dagger} \widetilde{M}_{\mathrm{D}}\right)_{13}=e^{\mathrm{i} \rho^{\mathrm{R}}}\left[A^{\prime} e^{\mathrm{i} \delta^{R}} s_{13}^{\mathrm{R}} c_{13}^{\mathrm{R}} c_{12}^{\mathrm{R} 2}-p e^{\mathrm{i} \delta^{R}} s_{13}^{\mathrm{R}} c_{12}^{\mathrm{R}}-t_{2} e^{\mathrm{i} \delta^{R}} s_{13}^{\mathrm{R}} c_{13}^{\mathrm{R}} c_{12}^{\mathrm{R}}\right. \\
& \left.-r^{*} s_{12}^{\mathrm{R}} c_{13}^{\mathrm{R}}+q c_{12}^{\mathrm{R}}-2 \mathrm{i} c_{12}^{\mathrm{R}} c_{13}^{\mathrm{R} 2} \operatorname{Im}(q)\right] .
\end{aligned}
$$

In view of eqs. (4.3) and (4.12) together with the definitions of $\eta, x$ and $y$, it is apparent that $p, t_{1}$ and $t_{2}$ are real; $q$ and $r$ are purely imaginary; $\mathrm{i} e^{\mathrm{i} \delta^{R}}= \pm 1, e^{\mathrm{i}\left(\rho^{\mathrm{R}}-\sigma^{\mathrm{R}}\right)}=1$ or $\pm \mathrm{i}$ 
and $e^{\mathrm{i} \rho^{\mathrm{R}}}=1$ or $\mathrm{i}$. We are therefore left with

$$
\operatorname{Im}\left[\left(\widetilde{M}_{\mathrm{D}}^{\dagger} \widetilde{M}_{\mathrm{D}}\right)_{12}^{2}\right]=\operatorname{Im}\left[\left(\widetilde{M}_{\mathrm{D}}^{\dagger} \widetilde{M}_{\mathrm{D}}\right)_{13}^{2}\right]=0, \quad \epsilon_{1}=0
$$

In other words, there is no $\mathrm{CP}$ violation at all in $N_{1}$ decays for category $A_{1}$.

If the three heavy Majorana neutrinos have the same mass hierarchy in categories $A_{1}$, $A_{2}$ and $A_{3}$, then the expressions of three eigenvalues of $M_{\mathrm{R}}^{A_{i}}$ are of the same form, and therefore eq. (2.16) leads us to

$$
U_{\mathrm{R}}^{A_{2}}=S^{(231)} U_{\mathrm{R}}^{A_{1}}, \quad U_{\mathrm{R}}^{A_{3}}=S^{(312)} U_{\mathrm{R}}^{A_{1}} .
$$

With the help of eqs. (2.16) and (4.15), we find

$$
\begin{aligned}
\widetilde{M}_{\mathrm{D}}^{A_{2} \dagger} \widetilde{M}_{\mathrm{D}}^{A_{2}} & =U_{\mathrm{R}}^{A_{2} T} M_{\mathrm{D}}^{A_{2} \dagger} M_{\mathrm{D}}^{A_{2}} U_{\mathrm{R}}^{A_{2} *} \\
& =U_{\mathrm{R}}^{A_{1} T} S^{(312)} S^{(231)} M_{\mathrm{D}}^{A_{1} \dagger} S^{(312)} S^{(231)} M_{\mathrm{D}}^{A_{1}} S^{(312)} S^{(231)} U_{\mathrm{R}}^{A_{1} *} \\
& =U_{\mathrm{R}}^{A_{1} T} M_{\mathrm{D}}^{A_{1} \dagger} M_{\mathrm{D}}^{A_{1}} U_{\mathrm{R}}^{A_{1} *} \\
& =\widetilde{M}_{\mathrm{D}}^{A_{1} \dagger} \widetilde{M}_{\mathrm{D}}^{A_{1}}, \\
\widetilde{M}_{\mathrm{D}}^{A_{3} \dagger} \widetilde{M}_{\mathrm{D}}^{A_{3}} & =U_{\mathrm{R}}^{A_{3} T} M_{\mathrm{D}}^{A_{3} \dagger} M_{\mathrm{D}}^{A_{3}} U_{\mathrm{R}}^{A_{3} *} \\
& =U_{\mathrm{R}}^{A_{1} T} S^{(231)} S^{(312)} M_{\mathrm{D}}^{A_{1} \dagger} S^{(231)} S^{(312)} M_{\mathrm{D}}^{A_{1}} S^{(231)} S^{(312)} U_{\mathrm{R}}^{A_{1} *} \\
& =U_{\mathrm{R}}^{A_{1} T} M_{\mathrm{D}}^{A_{1} \dagger} M_{\mathrm{D}}^{A_{1}} U_{\mathrm{R}}^{A_{1} *} \\
& =\widetilde{M}_{\mathrm{D}}^{A_{1} \dagger} \widetilde{M}_{\mathrm{D}}^{A_{1}} .
\end{aligned}
$$

This result in turn means

$$
\epsilon_{1}^{A_{2}}=\epsilon_{1}^{A_{3}}=\epsilon_{1}^{A_{1}}=0 .
$$

We conclude that in the $S_{3}$ reflection symmetry limit there is no way to realize unflavored leptogenesis for categories $A_{i}$. This conclusion will change when the lepton flavor effects are taken into account.

\subsubsection{Category $C$}

In this case the three eigenvalues of $M_{\mathrm{R}}$ are given by $a_{\mathrm{r}}-b_{\mathrm{r}}, a_{\mathrm{r}}-b_{\mathrm{r}}$ and $a_{\mathrm{r}}+2 b_{\mathrm{r}}$, respectively. For simplicity, let us assume $a_{\mathrm{r}}>0$ and $b_{\mathrm{r}}<0$, such that $M_{1}=a_{\mathrm{r}}+2 b_{\mathrm{r}} \ll M_{2}=M_{3}=$ $a_{\mathrm{r}}-b_{\mathrm{r}}$ can be satisfied. The corresponding unitary matrix $U_{\mathrm{R}}$ is

$$
U_{\mathrm{R}}=\left(\begin{array}{ccc}
\frac{1}{\sqrt{3}} & -\frac{2}{\sqrt{6}} & 0 \\
\frac{1}{\sqrt{3}} & \frac{1}{\sqrt{6}} & \frac{1}{\sqrt{2}} \\
\frac{1}{\sqrt{3}} & \frac{1}{\sqrt{6}} & -\frac{1}{\sqrt{2}}
\end{array}\right)
$$

Consequently,

$$
\widetilde{M}_{\mathrm{D}}=M_{\mathrm{D}} U_{\mathrm{R}}^{*}=\left(\begin{array}{ccc}
\frac{1}{\sqrt{3}}\left(A_{\mathrm{r}}+2 \operatorname{Re} B\right)-\frac{1}{\sqrt{6}}\left(2 A_{\mathrm{r}}-2 \operatorname{Re} B\right) & \sqrt{2} \mathrm{i} \operatorname{Im} B \\
\frac{1}{\sqrt{3}}\left(A_{\mathrm{r}}+2 \operatorname{Re} B\right) & \frac{1}{\sqrt{6}}\left(A_{\mathrm{r}}-2 B^{*}+B\right) & \frac{1}{\sqrt{2}}\left(A_{\mathrm{r}}-B\right) \\
\frac{1}{\sqrt{3}}\left(A_{\mathrm{r}}+2 \operatorname{Re} B\right) & \frac{1}{\sqrt{6}}\left(A_{\mathrm{r}}+B^{*}-2 B\right) & -\frac{1}{\sqrt{2}}\left(A_{\mathrm{r}}-B^{*}\right)
\end{array}\right) .
$$


Then it is straightforward for us to obtain

$$
\left(\widetilde{M}_{\mathrm{D}}^{\dagger} \widetilde{M}_{\mathrm{D}}\right)_{11}=\left(A_{\mathrm{r}}+2 \operatorname{Re} B\right)^{2}, \quad\left(\widetilde{M}_{\mathrm{D}}^{\dagger} \widetilde{M}_{\mathrm{D}}\right)_{12}=0, \quad\left(\widetilde{M}_{\mathrm{D}}^{\dagger} \widetilde{M}_{\mathrm{D}}\right)_{13}=0
$$

As a result,

$$
\epsilon_{1 \alpha}=0, \quad \epsilon_{1}=\sum_{\alpha} \epsilon_{1 \alpha}=0
$$

where $\alpha$ runs over $e, \mu$ and $\tau$. Therefore, there is no way for both unflavored and flavored leptogenesis to work in category $C$.

\subsection{Flavored leptogenesis}

In the unflavored leptogenesis case as discussed above, the Yukawa interactions of charged leptons are not taken into account, since the equilibrium temperature of heavy Majorana neutrinos is assumed to be high enough that such interactions cannot distinguish one lepton flavor from another. In other words, all the relevant Yukawa interactions are blind to lepton flavors. When the equilibrium temperature is lower, however, it is possible that the Yukawa interactions of charged leptons become faster than the (inverse) decays of $N_{i}$ or equivalently comparable to the expansion rate of the Universe. In this case the flavor effects must be taken into consideration [41, 42].

Here we focus on the possibility that the equilibrium temperature $T$ lies in the range $10^{9} \mathrm{GeV}<T<10^{12} \mathrm{GeV}$, in which the $\tau$ lepton can be in thermal equilibrium and thus are distinguishable from the $e$ and $\mu$ flavors. In this case both the CP-violating asymmetries and washout effects involving the $\tau$ flavor should be treated separately $[57,58]$. It is then possible to achieve successful leptogenesis provided $\epsilon_{1 \alpha} \neq 0$ holds, even though the total CP-violating asymmetry $\epsilon_{1}$ is vanishing or vanishingly small.

We have shown in eq. (4.21) that both $\epsilon_{1 \alpha}$ and $\epsilon_{1}$ are vanishing in category $C$, and thus it is impossible to realize either unflavored or flavored leptogenesis in this case in the $S_{3}$ reflection symmetry limit. In the following we calculate the flavor-dependent CPviolating asymmetries $\epsilon_{1 \alpha}$ for categories $A_{i}$ by using eq. (4.1), to examine whether flavored leptogenesis has a chance to work or not in this case.

Given $M_{\mathrm{D}}$ and $U_{\mathrm{R}}$ in category $A_{1}$, a lengthy but straightforward calculation leads us to

$$
\begin{aligned}
\left(\widetilde{M}_{\mathrm{D}}^{*}\right)_{e 1}\left(\widetilde{M}_{\mathrm{D}}\right)_{e 2}= & e^{\mathrm{i}\left(\rho^{\mathrm{R}}-\sigma^{\mathrm{R}}\right)}\left\{A_{\mathrm{r}}^{2} s_{12}^{\mathrm{R}} c_{12}^{\mathrm{R}} c_{13}^{\mathrm{R} 2}+\sqrt{2} A_{\mathrm{r}} c_{13}^{\mathrm{R}} \cos 2 \theta_{12}^{\mathrm{R}} \operatorname{Re}\left(\eta^{*} B e^{-\mathrm{i} \phi_{2}^{\mathrm{R}}}\right)\right. \\
& +2 \mathrm{i} e^{\mathrm{i} \delta^{\mathrm{R}}} s_{13}^{\mathrm{R}} \cos 2 \theta_{12}^{\mathrm{R}} \operatorname{Re}\left(\eta^{*} B e^{-\mathrm{i} \phi_{2}^{\mathrm{R}}}\right) \operatorname{Im}\left(\eta^{*} B e^{-\mathrm{i} \phi_{2}^{\mathrm{R}}}\right) \\
& +2 s_{13}^{\mathrm{R} 2} s_{12}^{\mathrm{R}} c_{12}^{\mathrm{R}}\left[\operatorname{Im}\left(\eta^{*} B e^{-\mathrm{i} \phi_{2}^{\mathrm{R}}}\right)\right]^{2}-2 s_{12}^{\mathrm{R}} c_{12}^{\mathrm{R}}\left[\operatorname{Re}\left(\eta^{*} B e^{-\mathrm{i} \phi_{2}^{\mathrm{R}}}\right)\right]^{2} \\
+ & \left.2 \sqrt{2} \mathrm{i} e^{\mathrm{i} \delta^{\mathrm{R}}} A_{\mathrm{r}} s_{13}^{\mathrm{R}} c_{13}^{\mathrm{R}} s_{12}^{\mathrm{R}} c_{12}^{\mathrm{R}} \operatorname{Im}\left(\eta^{*} B e^{-\mathrm{i} \phi_{2}^{\mathrm{R}}}\right)\right\} \\
\left(\widetilde{M}_{\mathrm{D}}^{*}\right)_{e 1}\left(\widetilde{M}_{\mathrm{D}}\right)_{e 3}= & e^{\mathrm{i} \rho^{\mathrm{R}}}\left\{A_{\mathrm{r}}^{2} s_{13}^{\mathrm{R}} c_{13}^{\mathrm{R}} c_{12}^{\mathrm{R}} e^{\mathrm{i} \delta^{\mathrm{R}}}-2 e^{\mathrm{i} \delta^{\mathrm{R}}} s_{13}^{\mathrm{R}} c_{13}^{\mathrm{R}} c_{12}^{\mathrm{R}}\left[\operatorname{Im}\left(\eta^{*} B e^{-\mathrm{i} \phi_{2}^{\mathrm{R}}}\right)\right]^{2}\right. \\
& -2 \mathrm{i} s_{12}^{\mathrm{R}} c_{13}^{\mathrm{R}} \operatorname{Re}\left(\eta^{*} B e^{-\mathrm{i} \phi_{2}^{\mathrm{R}}}\right) \operatorname{Im}\left(\eta^{*} B e^{-\mathrm{i} \phi_{2}^{\mathrm{R}}}\right)
\end{aligned}
$$




$$
\begin{aligned}
& +\sqrt{2} \mathrm{i} A_{\mathrm{r}} \cos 2 \theta_{13}^{\mathrm{R}} c_{12}^{\mathrm{R}} \operatorname{Im}\left(\eta^{*} B e^{-\mathrm{i} \phi_{2}^{\mathrm{R}}}\right) \\
& \left.-\sqrt{2} A_{\mathrm{r}} s_{13}^{\mathrm{R}} s_{12}^{\mathrm{R}} e^{\mathrm{i} \delta^{\mathrm{R}}} \operatorname{Re}\left(\eta^{*} B e^{-\mathrm{i} \phi_{2}^{\mathrm{R}}}\right)\right\},
\end{aligned}
$$

and

$$
\begin{aligned}
\left(\widetilde{M}_{\mathrm{D}}^{*}\right)_{\mu 1}\left(\widetilde{M}_{\mathrm{D}}\right)_{\mu 2}= & e^{\mathrm{i}\left(\rho^{\mathrm{R}}-\sigma^{\mathrm{R}}\right)}\left\{|E|^{2} s_{12}^{\mathrm{R}} c_{12}^{\mathrm{R}} c_{13}^{\mathrm{R} 2}-\frac{1}{2}\left|z_{1}+z_{2}\right|^{2} s_{12}^{\mathrm{R}} c_{12}^{\mathrm{R}}+\frac{1}{2}\left|z_{1}-z_{2}\right|^{2} s_{13}^{\mathrm{R} 2} s_{12}^{\mathrm{R}} c_{12}^{\mathrm{R}}\right. \\
& -\sqrt{2} s_{12}^{\mathrm{R} 2} c_{13}^{\mathrm{R}} \operatorname{Re}\left[E^{*}\left(z_{1}+z_{2}\right)\right]+\sqrt{2} \mathrm{i} e^{\mathrm{i} \delta^{\mathrm{R}}} s_{13}^{\mathrm{R}} c_{13}^{\mathrm{R}} s_{12}^{\mathrm{R}} c_{12}^{\mathrm{R}} \operatorname{Im}\left[E^{*}\left(z_{1}-z_{2}\right)\right] \\
& -\mathrm{i} e^{\mathrm{i} \delta^{\mathrm{R}}} s_{13}^{\mathrm{R}} \cos 2 \theta_{12}^{\mathrm{R}} \operatorname{Im}\left(z_{1}^{*} z_{2}\right)+\frac{1}{\sqrt{2}} c_{13}^{\mathrm{R}} E^{*}\left(z_{1}+z_{2}\right) \\
& \left.-\frac{1}{2} s_{13}^{\mathrm{R}} e^{\mathrm{i} \delta^{\mathrm{R}}}\left(|C|^{2}-|D|^{2}\right)\right\}, \\
\left(\widetilde{M}_{\mathrm{D}}^{*}\right)_{\mu 1}\left(\widetilde{M}_{\mathrm{D}}\right)_{\mu 3}= & e^{\mathrm{i} \rho^{\mathrm{R}}}\left\{|E|^{2} s_{13}^{\mathrm{R}} c_{13}^{\mathrm{R}} c_{12}^{\mathrm{R}} e^{\mathrm{i} \delta^{\mathrm{R}}}-\frac{1}{2} s_{12}^{\mathrm{R}} c_{13}^{\mathrm{R}}\left(|C|^{2}-|D|^{2}\right)+\mathrm{i} s_{12}^{\mathrm{R}} c_{13}^{\mathrm{R}} \operatorname{Im}\left(z_{1}^{*} z_{2}\right)\right. \\
& -\sqrt{2} \mathrm{i} s_{13}^{\mathrm{R} 2} c_{12}^{\mathrm{R}} \operatorname{Im}\left[E^{*}\left(z_{1}-z_{2}\right)\right]-\frac{1}{2} e^{\mathrm{i} \delta^{\mathrm{R}}} s_{13}^{\mathrm{R}} c_{13}^{\mathrm{R}} c_{12}^{\mathrm{R}}\left|z_{1}-z_{2}\right|^{2} \\
& \left.+\frac{1}{\sqrt{2}} c_{12}^{\mathrm{R}} E^{*}\left(z_{1}-z_{2}\right)-\frac{1}{\sqrt{2}} e^{\mathrm{i} \delta^{\mathrm{R}}} s_{13}^{\mathrm{R}} s_{12}^{\mathrm{R}} E\left(z_{1}^{*}+z_{2}^{*}\right)\right\},
\end{aligned}
$$

as well as

$$
\begin{aligned}
& \left(\widetilde{M}_{\mathrm{D}}^{*}\right)_{\tau 1}\left(\widetilde{M}_{\mathrm{D}}\right)_{\tau 2}=e^{\mathrm{i}\left(\rho^{\mathrm{R}}-\sigma^{\mathrm{R}}\right)}\left\{|E|^{2} s_{12}^{\mathrm{R}} c_{12}^{\mathrm{R}} c_{13}^{\mathrm{R} 2}-\frac{1}{2}\left|z_{1}+z_{2}\right|^{2} s_{12}^{\mathrm{R}} c_{12}^{\mathrm{R}}+\frac{1}{2}\left|z_{1}-z_{2}\right|^{2} s_{13}^{\mathrm{R} 2} s_{12}^{\mathrm{R}} c_{12}^{\mathrm{R}}\right. \\
& -\sqrt{2} s_{12}^{\mathrm{R} 2} c_{13}^{\mathrm{R}} \operatorname{Re}\left[E^{*}\left(z_{1}+z_{2}\right)\right]+\sqrt{2} \mathrm{i} e^{\mathrm{i} \delta^{\mathrm{R}}} s_{13}^{\mathrm{R}} c_{13}^{\mathrm{R}} s_{12}^{\mathrm{R}} c_{12}^{\mathrm{R}} \operatorname{Im}\left[E^{*}\left(z_{1}-z_{2}\right)\right] \\
& -\mathrm{i} e^{\mathrm{i} \delta^{\mathrm{R}}} s_{13}^{\mathrm{R}} \cos 2 \theta_{12}^{\mathrm{R}} \operatorname{Im}\left(z_{1}^{*} z_{2}\right)+\frac{1}{2} s_{13}^{\mathrm{R}} e^{\mathrm{i} \delta^{\mathrm{R}}}\left(|C|^{2}-|D|^{2}\right) \\
& \left.+\frac{1}{\sqrt{2}} c_{13}^{\mathrm{R}} E\left(z_{1}^{*}+z_{2}^{*}\right)\right\} \text {, } \\
& \left(\widetilde{M}_{\mathrm{D}}^{*}\right)_{\tau 1}\left(\widetilde{M}_{\mathrm{D}}\right)_{\tau 3}=e^{\mathrm{i} \rho^{\mathrm{R}}}\left\{|E|^{2} s_{13}^{\mathrm{R}} c_{13}^{\mathrm{R}} c_{12}^{\mathrm{R}} e^{\mathrm{i} \delta^{\mathrm{R}}}+\frac{1}{2} s_{12}^{\mathrm{R}} c_{13}^{\mathrm{R}}\left(|C|^{2}-|D|^{2}\right)+\mathrm{i} s_{12}^{\mathrm{R}} c_{13}^{\mathrm{R}} \operatorname{Im}\left(z_{1}^{*} z_{2}\right)\right. \\
& -\sqrt{2} \text { i } s_{13}^{\mathrm{R} 2} c_{12}^{\mathrm{R}} \operatorname{Im}\left[E^{*}\left(z_{1}-z_{2}\right)\right]-\frac{1}{2} e^{\mathrm{i} \delta^{\mathrm{R}}} s_{13}^{\mathrm{R}} c_{13}^{\mathrm{R}} c_{12}^{\mathrm{R}}\left|z_{1}-z_{2}\right|^{2} \\
& \left.-\frac{1}{\sqrt{2}} c_{12}^{\mathrm{R}} E\left(z_{1}^{*}-z_{2}^{*}\right)-\frac{1}{\sqrt{2}} e^{\mathrm{i} \delta^{\mathrm{R}}} s_{13}^{\mathrm{R}} s_{12}^{\mathrm{R}} E^{*}\left(z_{1}+z_{2}\right)\right\},
\end{aligned}
$$

where $\eta=1$ (or $-\mathrm{i}$ ) for $\phi_{1}^{\mathrm{R}}=0($ or $\pi / 2)$, and

$$
z_{1}=\eta^{*} C e^{-\mathrm{i} \phi_{2}^{\mathrm{R}}}, \quad z_{2}=\eta D e^{\mathrm{i} \phi_{2}^{\mathrm{R}}} .
$$

With the help of eq. (4.13) and eqs. (4.22)-(4.25), we further obtain

$$
\begin{aligned}
& \operatorname{Im}\left[\left(\widetilde{M}_{\mathrm{D}}^{*}\right)_{e 1}\left(\widetilde{M}_{\mathrm{D}}\right)_{e 2}\left(\widetilde{M}_{\mathrm{D}}^{\dagger} \widetilde{M}_{\mathrm{D}}\right)_{12}\right]=0, \\
& \operatorname{Im}\left[\left(\widetilde{M}_{\mathrm{D}}^{*}\right)_{e 1}\left(\widetilde{M}_{\mathrm{D}}\right)_{e 3}\left(\widetilde{M}_{\mathrm{D}}^{\dagger} \widetilde{M}_{\mathrm{D}}\right)_{13}\right]=0, \\
& \operatorname{Im}\left[\left(\widetilde{M}_{\mathrm{D}}^{*}\right)_{\mu 1}\left(\widetilde{M}_{\mathrm{D}}\right)_{\mu 2}\left(\widetilde{M}_{\mathrm{D}}^{\dagger} \widetilde{M}_{\mathrm{D}}\right)_{12}\right]=\eta_{1}\left|\left(\widetilde{M}_{\mathrm{D}}^{\dagger} \widetilde{M}_{\mathrm{D}}\right)_{12}\right| \operatorname{Im}\left[\frac{1}{\sqrt{2}} c_{13}^{\mathrm{R}} E^{*}\left(z_{1}+z_{2}\right)\right. \\
& \left.\quad-\frac{1}{2} s_{13}^{\mathrm{R}} e^{\mathrm{i} \delta^{\mathrm{R}}}\left(|C|^{2}-|D|^{2}\right)\right],
\end{aligned}
$$




$$
\begin{aligned}
& \operatorname{Im}\left[\left(\widetilde{M}_{\mathrm{D}}^{*}\right)_{\mu 1}\left(\widetilde{M}_{\mathrm{D}}\right)_{\mu 3}\left(\widetilde{M}_{\mathrm{D}}^{\dagger} \widetilde{M}_{\mathrm{D}}\right)_{13}\right]=\eta_{2}\left|\left(\widetilde{M}_{\mathrm{D}}^{\dagger} \widetilde{M}_{\mathrm{D}}\right)_{13}\right| \operatorname{Re}\left[\frac{1}{\sqrt{2}} c_{12}^{\mathrm{R}} E^{*}\left(z_{1}-z_{2}\right)\right. \\
& \left.-\frac{1}{\sqrt{2}} e^{\mathrm{i} \delta^{\mathrm{R}}} s_{13}^{\mathrm{R}} s_{12}^{\mathrm{R}} E\left(z_{1}^{*}+z_{2}^{*}\right)-\frac{1}{2} s_{12}^{\mathrm{R}} c_{13}^{\mathrm{R}}\left(|C|^{2}-|D|^{2}\right)\right], \\
& \operatorname{Im}\left[\left(\widetilde{M}_{\mathrm{D}}^{*}\right)_{\tau 1}\left(\widetilde{M}_{\mathrm{D}}\right)_{\tau 2}\left(\widetilde{M}_{\mathrm{D}}^{\dagger} \widetilde{M}_{\mathrm{D}}\right)_{12}\right]=\eta_{1}\left|\left(\widetilde{M}_{\mathrm{D}}^{\dagger} \widetilde{M}_{\mathrm{D}}\right)_{12}\right| \operatorname{Im}\left[\frac{1}{\sqrt{2}} c_{13}^{\mathrm{R}} E\left(z_{1}^{*}+z_{2}^{*}\right)\right. \\
& \left.+\frac{1}{2} s_{13}^{\mathrm{R}} e^{\mathrm{i} \delta^{\mathrm{R}}}\left(|C|^{2}-|D|^{2}\right)\right], \\
& \operatorname{Im}\left[\left(\widetilde{M}_{\mathrm{D}}^{*}\right)_{\tau 1}\left(\widetilde{M}_{\mathrm{D}}\right)_{\tau 3}\left(\widetilde{M}_{\mathrm{D}}^{\dagger} \widetilde{M}_{\mathrm{D}}\right)_{13}\right]=\eta_{2}\left|\left(\widetilde{M}_{\mathrm{D}}^{\dagger} \widetilde{M}_{\mathrm{D}}\right)_{13}\right| \operatorname{Re}\left[-\frac{1}{\sqrt{2}} c_{12}^{\mathrm{R}} E\left(z_{1}^{*}-z_{2}^{*}\right)\right. \\
& -\frac{1}{\sqrt{2}} e^{\mathrm{i} \delta^{\mathrm{R}}} s_{13}^{\mathrm{R}} s_{12}^{\mathrm{R}} E^{*}\left(z_{1}+z_{2}\right) \\
& \left.+\frac{1}{2} s_{12}^{\mathrm{R}} c_{13}^{\mathrm{R}}\left(|C|^{2}-|D|^{2}\right)\right] \text {, }
\end{aligned}
$$

and

$$
\begin{aligned}
& \operatorname{Im}\left[\left(\widetilde{M}_{\mathrm{D}}^{*}\right)_{e 1}\left(\widetilde{M}_{\mathrm{D}}\right)_{e 2}\left(\widetilde{M}_{\mathrm{D}}^{\dagger} \widetilde{M}_{\mathrm{D}}\right)_{12}^{*}\right]=0, \\
& \operatorname{Im}\left[\left(\widetilde{M}_{\mathrm{D}}^{*}\right)_{e 1}\left(\widetilde{M}_{\mathrm{D}}\right)_{e 3}\left(\widetilde{M}_{\mathrm{D}}^{\dagger} \widetilde{M}_{\mathrm{D}}\right)_{13}^{*}\right]=0, \\
& \operatorname{Im}\left[\left(\widetilde{M}_{\mathrm{D}}^{*}\right)_{\mu 1}\left(\widetilde{M}_{\mathrm{D}}\right)_{\mu 2}\left(\widetilde{M}_{\mathrm{D}}^{\dagger} \widetilde{M}_{\mathrm{D}}\right)_{12}^{*}\right]=\eta_{3}\left|\left(\widetilde{M}_{\mathrm{D}}^{\dagger} \widetilde{M}_{\mathrm{D}}\right)_{12}\right| \operatorname{Im}\left[\frac{1}{\sqrt{2}} c_{13}^{\mathrm{R}} E^{*}\left(z_{1}+z_{2}\right)\right. \\
& \left.-\frac{1}{2} s_{13}^{\mathrm{R}} e^{\mathrm{i} \delta^{\mathrm{R}}}\left(|C|^{2}-|D|^{2}\right)\right], \\
& \operatorname{Im}\left[\left(\widetilde{M}_{\mathrm{D}}^{*}\right)_{\mu 1}\left(\widetilde{M}_{\mathrm{D}}\right)_{\mu 3}\left(\widetilde{M}_{\mathrm{D}}^{\dagger} \widetilde{M}_{\mathrm{D}}\right)_{13}^{*}\right]=\eta_{4}\left|\left(\widetilde{M}_{\mathrm{D}}^{\dagger} \widetilde{M}_{\mathrm{D}}\right)_{13}\right| \operatorname{Re}\left[\frac{1}{\sqrt{2}} c_{12}^{\mathrm{R}} E^{*}\left(z_{1}-z_{2}\right)\right. \\
& \left.-\frac{1}{\sqrt{2}} e^{\mathrm{i} \delta^{\mathrm{R}}} s_{13}^{\mathrm{R}} s_{12}^{\mathrm{R}} E\left(z_{1}^{*}+z_{2}^{*}\right)-\frac{1}{2} s_{12}^{\mathrm{R}} c_{13}^{\mathrm{R}}\left(|C|^{2}-|D|^{2}\right)\right], \\
& \operatorname{Im}\left[\left(\widetilde{M}_{\mathrm{D}}^{*}\right)_{\tau 1}\left(\widetilde{M}_{\mathrm{D}}\right)_{\tau 2}\left(\widetilde{M}_{\mathrm{D}}^{\dagger} \widetilde{M}_{\mathrm{D}}\right)_{12}^{*}\right]=\eta_{3}\left|\left(\widetilde{M}_{\mathrm{D}}^{\dagger} \widetilde{M}_{\mathrm{D}}\right)_{12}\right| \operatorname{Im}\left[\frac{1}{\sqrt{2}} c_{13}^{\mathrm{R}} E\left(z_{1}^{*}+z_{2}^{*}\right)\right. \\
& \left.+\frac{1}{2} s_{13}^{\mathrm{R}} e^{\mathrm{i} \delta^{\mathrm{R}}}\left(|C|^{2}-|D|^{2}\right)\right], \\
& \operatorname{Im}\left[\left(\widetilde{M}_{\mathrm{D}}^{*}\right)_{\tau 1}\left(\widetilde{M}_{\mathrm{D}}\right)_{\tau 3}\left(\widetilde{M}_{\mathrm{D}}^{\dagger} \widetilde{M}_{\mathrm{D}}\right)_{13}^{*}\right]=\eta_{4}\left|\left(\widetilde{M}_{\mathrm{D}}^{\dagger} \widetilde{M}_{\mathrm{D}}\right)_{13}\right| \operatorname{Re}\left[-\frac{1}{\sqrt{2}} c_{12}^{\mathrm{R}} E\left(z_{1}^{*}-z_{2}^{*}\right)\right. \\
& -\frac{1}{\sqrt{2}} e^{\mathrm{i} \delta^{\mathrm{R}}} s_{13}^{\mathrm{R}} s_{12}^{\mathrm{R}} E^{*}\left(z_{1}+z_{2}\right) \\
& \left.+\frac{1}{2} s_{12}^{\mathrm{R}} c_{13}^{\mathrm{R}}\left(|C|^{2}-|D|^{2}\right)\right],
\end{aligned}
$$

where $\eta_{i}= \pm 1$ (for $\left.i=1,2,3,4\right)$. Combining eqs. (4.26) and (4.27), we arrive at

$$
\begin{aligned}
& \operatorname{Im}\left[\left(\widetilde{M}_{\mathrm{D}}^{*}\right)_{\mu 1}\left(\widetilde{M}_{\mathrm{D}}\right)_{\mu 2}\left(\widetilde{M}_{\mathrm{D}}^{\dagger} \widetilde{M}_{\mathrm{D}}\right)_{12}\right]=-\operatorname{Im}\left[\left(\widetilde{M}_{\mathrm{D}}^{*}\right)_{\tau 1}\left(\widetilde{M}_{\mathrm{D}}\right)_{\tau 2}\left(\widetilde{M}_{\mathrm{D}}^{\dagger} \widetilde{M}_{\mathrm{D}}\right)_{12}\right] \neq 0, \\
& \operatorname{Im}\left[\left(\widetilde{M}_{\mathrm{D}}^{*}\right)_{\mu 1}\left(\widetilde{M}_{\mathrm{D}}\right)_{\mu 3}\left(\widetilde{M}_{\mathrm{D}}^{\dagger} \widetilde{M}_{\mathrm{D}}\right)_{13}\right]=-\operatorname{Im}\left[\left(\widetilde{M}_{\mathrm{D}}^{*}\right)_{\tau 1}\left(\widetilde{M}_{\mathrm{D}}\right)_{\tau 3}\left(\widetilde{M}_{\mathrm{D}}^{\dagger} \widetilde{M}_{\mathrm{D}}\right)_{13}\right] \neq 0,
\end{aligned}
$$




$$
\begin{aligned}
& \operatorname{Im}\left[\left(\widetilde{M}_{\mathrm{D}}^{*}\right)_{\mu 1}\left(\widetilde{M}_{\mathrm{D}}\right)_{\mu 2}\left(\widetilde{M}_{\mathrm{D}}^{\dagger} \widetilde{M}_{\mathrm{D}}\right)_{12}^{*}\right]=-\operatorname{Im}\left[\left(\widetilde{M}_{\mathrm{D}}^{*}\right)_{\tau 1}\left(\widetilde{M}_{\mathrm{D}}\right)_{\tau 2}\left(\widetilde{M}_{\mathrm{D}}^{\dagger} \widetilde{M}_{\mathrm{D}}\right)_{12}^{*}\right] \neq 0 \\
& \operatorname{Im}\left[\left(\widetilde{M}_{\mathrm{D}}^{*}\right)_{\mu 1}\left(\widetilde{M}_{\mathrm{D}}\right)_{\mu 3}\left(\widetilde{M}_{\mathrm{D}}^{\dagger} \widetilde{M}_{\mathrm{D}}\right)_{13}^{*}\right]=-\operatorname{Im}\left[\left(\widetilde{M}_{\mathrm{D}}^{*}\right)_{\tau 1}\left(\widetilde{M}_{\mathrm{D}}\right)_{\tau 3}\left(\widetilde{M}_{\mathrm{D}}^{\dagger} \widetilde{M}_{\mathrm{D}}\right)_{13}^{*}\right] \neq 0 .
\end{aligned}
$$

As a result,

$$
\epsilon_{1 e}=0, \quad \epsilon_{1 \mu}=-\epsilon_{1 \tau} \neq 0, \quad \epsilon_{1}=\sum_{\alpha} \epsilon_{1 \alpha}=0 .
$$

It is therefore possible to realize $\mu$ - or $\tau$-flavored leptogenesis in this case, at least in principle. Since a realistic example of this kind needs to include proper $S_{3}$ reflection symmetry breaking effects, it will be studied elsewhere.

Finally, if the mass hierarchies of three heavy Majorana neutrinos in categories $A_{2}$ and $A_{3}$ are the same as that in category $A_{1}$, then one can get

$$
\begin{aligned}
& \left(\widetilde{M}_{\mathrm{D}}^{A_{2} *}\right)_{\alpha 1}\left(\widetilde{M}_{\mathrm{D}}^{A_{2}}\right)_{\alpha j}=\left(\widetilde{M}_{\mathrm{D}}^{A_{1} *}\right)_{\beta 1}\left(\widetilde{M}_{\mathrm{D}}^{A_{1}}\right)_{\beta j}, \\
& \left(\widetilde{M}_{\mathrm{D}}^{A_{3} *}\right)_{\gamma 1}\left(\widetilde{M}_{\mathrm{D}}^{A_{3}}\right)_{\gamma j}=\left(\widetilde{M}_{\mathrm{D}}^{A_{1} *}\right)_{\lambda 1}\left(\widetilde{M}_{\mathrm{D}}^{A_{1}}\right)_{\lambda j},
\end{aligned}
$$

and therefore

$$
\epsilon_{1 \alpha}^{A_{2}}=\epsilon_{1 \beta}^{A_{1}}, \quad \epsilon_{1 \gamma}^{A_{3}}=\epsilon_{1 \lambda}^{A_{1}},
$$

where $\alpha \beta=e \mu, \mu \tau$ and $\tau e ; \gamma \lambda=e \tau, \mu e$ and $\tau \mu$; and $j=2$ or 3 . As a result,

$$
\begin{array}{lll}
\epsilon_{1 \tau}^{A_{2}}=0, & \epsilon_{1 e}^{A_{2}}=-\epsilon_{1 \mu}^{A_{2}} \neq 0, & \epsilon_{1}^{A_{2}}=\sum_{\alpha} \epsilon_{1 \alpha}^{A_{2}}=0 ; \\
\epsilon_{1 \mu}^{A_{3}}=0, & \epsilon_{1 \tau}^{A_{3}}=-\epsilon_{1 e}^{A_{3}} \neq 0, & \epsilon_{1}^{A_{3}}=\sum_{\alpha} \epsilon_{1 \alpha}^{A_{3}}=0
\end{array}
$$

for categories $A_{2}$ and $A_{3}$.

\section{Some further discussions}

In this work we have made a new attempt to specify the flavor structures associated with the canonical seesaw mechanism, so as to promote its predictability and testability. What we have done is to require the relevant neutrino mass terms to be invariant under the $S_{3}$ reflection transformations of both left- and right-handed neutrino fields. This treatment allows us to constrain the Dirac mass matrix $M_{\mathrm{D}}$ and the right-handed neutrino mass matrix $M_{\mathrm{R}}$ to some extent, and the effective light Majorana neutrino mass matrix $M_{\nu}$ is in turn constrained through the seesaw relation. We find that the structures of $M_{\mathrm{D}}$, $M_{\mathrm{R}}$ and $M_{\nu}$ can be classified into 22 categories, among which some structures respect the well-known $\mu-\tau$ symmetry and (or) flavor democracy. In particular, we find that the texture of $M_{\nu}$ may be either the same as or similar to that of $M_{\mathrm{R}}$, and this property reflects a seesaw mirroring relationship between light and heavy Majorana neutrinos. To be specific, we have calculated the light neutrino masses and flavor mixing parameters for all the textures of $M_{\nu}$, and examined whether the CP-violating asymmetries in decays of 
the lightest heavy Majorana neutrino are vanishing or not in the $S_{3}$ reflection symmetry limit. Our calculations show that only the flavored leptogenesis mechanism is possible to work for categories $A_{1}, A_{2}$ and $A_{3}$ listed in table 1 .

One might wonder whether some different neutrino mixing patterns and related leptogenesis can be obtained in our approach if $S_{3}$ symmetry group is extended to $S_{4}$ or $A_{4}$. The answer is affirmative. To illustrate, let us briefly discuss the situation associated with $A_{4}$ group in our framework. It is well known that $A_{4}$ group is defined as the even permutation of four objects and has twelve elements being divided into four classes. So $A_{4}$ group has four irreducible representations - three inequivalent one-dimensional representations $(\underline{\mathbf{1}}$, $\underline{\mathbf{1}}^{\prime}$ and $\underline{\mathbf{1}}^{\prime \prime}$ ) and one three-dimensional representation $(\underline{\mathbf{3}})$. Now that we work in the basis where $M_{l}$ is diagonal, it is more interesting for us to consider the three-dimensional unitary representation of $A_{4}$ group, which has been used in refs. [8, 59] rather than refs. [60, 61]. In this representation the two generators of $A_{4}$, denoted as $S$ and $T$, are given by

$$
S=\frac{1}{3}\left(\begin{array}{ccc}
-1 & 2 & 2 \\
2 & -1 & 2 \\
2 & 2 & -1
\end{array}\right), \quad T=\left(\begin{array}{ccc}
1 & 0 & 0 \\
0 & \omega^{2} & 0 \\
0 & 0 & \omega
\end{array}\right)
$$

where $\omega=\exp (\mathrm{i} 2 \pi / 3)$. Then all the elements of $A_{4}$ can be presented by the following twelve $3 \times 3$ matrices: $\mathbf{1}, S, T, S T, T S, T^{2}, S T^{2}, T^{2} S, S T S, T S T, T S T^{2}$ and $T^{2} S T$.

In a way similar to the $S_{3}$ reflection transformations, we may require the neutrino mass term in eq. (2.1) to keep invariant under the transformations made in eq. (2.2) with $S_{(\mathrm{L})}$ or $S_{(\mathrm{R})}$ being an arbitrary element of the given subset of $A_{4}$ group. In this case we are left with the same form of the constraints on $M_{\mathrm{D}}$ and $M_{\mathrm{R}}$ as obtained in eq. (2.5). One may systematically categorize all the possible structures of neutrino mass matrices as we have done in table 1 for $S_{3}$ group, but for $A_{4}$ group it seems unnecessary to do so because in most cases the $A_{4}$-induced constraints are so strong that the resultant textures of neutrino mass matrices are trivial and disinteresting. If a case with the given subset having more than one element is considered, for example, it will be unable to result in any $\mathrm{CP}$ violation in both light and heavy neutrino sectors. Some cases with only one element may also lead to trivial results, and those more interesting cases usually involve many unknown parameters. Here we only show a simple example of this kind which allows us to obtain the textures of neutrino mass matrices different from those listed in table 1 , together with a nonzero CPviolating asymmetry $\epsilon_{1}$ in the lightest heavy Majorana neutrino decays. It is the case where the subset only contains element $S$, and in this case $M_{\mathrm{D}}$ and $M_{\mathrm{R}}$ constrained by eq. (2.5) are

$$
\begin{aligned}
M_{\mathrm{D}} & =\left(\begin{array}{ccc}
2 A_{\mathrm{r}} & 2 B_{\mathrm{r}} & 2 B_{\mathrm{r}} \\
2 B_{\mathrm{r}} & A_{\mathrm{r}}+B_{\mathrm{r}} & A_{\mathrm{r}}+B_{\mathrm{r}} \\
2 B_{\mathrm{r}} & A_{\mathrm{r}}+B_{\mathrm{r}} & A_{\mathrm{r}}+B_{\mathrm{r}}
\end{array}\right)+\mathrm{i} C_{\mathrm{r}}\left(\begin{array}{ccc}
2 & 2 & 2 \\
-1 & -1 & -1 \\
-1 & -1 & -1
\end{array}\right), \\
M_{\mathrm{R}} & =\left(\begin{array}{ccc}
a_{\mathrm{r}} & b_{\mathrm{r}} & e_{\mathrm{r}} \\
b_{\mathrm{r}} & d_{\mathrm{r}} & a_{\mathrm{r}}+e_{\mathrm{r}}-d_{\mathrm{r}} \\
e_{\mathrm{r}} & a_{\mathrm{r}}+e_{\mathrm{r}}-d_{\mathrm{r}} & b_{\mathrm{r}}-e_{\mathrm{r}}+d_{\mathrm{r}}
\end{array}\right),
\end{aligned}
$$

where $\operatorname{Re}\left[\left\langle M_{\mathrm{D}}\right\rangle_{12}\right]=\operatorname{Re}\left[\left\langle M_{\mathrm{D}}\right\rangle_{13}\right]=\operatorname{Re}\left[\left\langle M_{\mathrm{D}}\right\rangle_{21}\right], \quad \operatorname{Re}\left[\left\langle M_{\mathrm{D}}\right\rangle_{22}\right]=$ $\left(\operatorname{Re}\left[\left\langle M_{\mathrm{D}}\right\rangle_{11}\right]+\operatorname{Re}\left[\left\langle M_{\mathrm{D}}\right\rangle_{12}\right]\right) / 2, \quad \operatorname{Im}\left[\left\langle M_{\mathrm{D}}\right\rangle_{11}\right]=\operatorname{Im}\left[\left\langle M_{\mathrm{D}}\right\rangle_{12}\right]=\operatorname{Im}\left[\left\langle M_{\mathrm{D}}\right\rangle_{13}\right]$, 
$\operatorname{Im}\left[\left\langle M_{\mathrm{D}}\right\rangle_{21}\right]=-\operatorname{Im}\left[\left\langle M_{\mathrm{D}}\right\rangle_{11}\right] / 2$ and $\operatorname{Im}\left[M_{\mathrm{R}}\right]=0$ have been assumed to reduce the number of free parameters. The light Majorana neutrino mass matrix $M_{\nu}$ turns out to have the form

$$
M_{\nu}=\left(\begin{array}{cccc}
2 a_{\mathrm{r}}^{\prime}-b_{\mathrm{r}}^{\prime} & b_{\mathrm{r}}^{\prime} & b_{\mathrm{r}}^{\prime} \\
b_{\mathrm{r}}^{\prime} & a_{\mathrm{r}}^{\prime} & a_{\mathrm{r}}^{\prime} \\
b_{\mathrm{r}}^{\prime} & a_{\mathrm{r}}^{\prime} & a_{\mathrm{r}}^{\prime}
\end{array}\right)+\mathrm{i} e_{\mathrm{r}}^{\prime}\left(\begin{array}{ccc}
4 & 1 & 1 \\
1 & -2 & -2 \\
1 & -2 & -2
\end{array}\right),
$$

and the explicit relations between the parameters of $M_{\nu}$ and those of $M_{\mathrm{D}}$ and $M_{\mathrm{R}}$ can easily be derived. We see that this example leads to a texture of $M_{\nu}$ which respects the $\mu-\tau$ permutation symmetry. Diagonalizing $M_{\nu}$ allows us to obtain the masses of three light neutrinos and their flavor mixing parameters. For the inverted neutrino mass hierarchy, ${ }^{5}$ the results are

$$
m_{1}=\frac{1}{2}\left(-3 b_{\mathrm{r}}^{\prime}+\Delta\right), \quad m_{2}=\frac{1}{2}\left(3 b_{\mathrm{r}}^{\prime}+\Delta\right), \quad m_{3}=0
$$

and

$$
V=\left(\begin{array}{llcc} 
\pm \frac{2}{\sqrt{6}} \frac{-4 a_{\mathrm{r}}^{\prime}+b_{\mathrm{r}}^{\prime}-\Delta-6 \mathrm{i} e_{\mathrm{r}}^{\prime}}{t} & \pm \frac{1}{\sqrt{3}} \frac{4 a_{\mathrm{r}}^{\prime}-b_{\mathrm{r}}^{\prime}+\Delta+12 \mathrm{i} e_{\mathrm{r}}^{\prime}}{t} & 0 \\
\pm \frac{1}{\sqrt{6}} \frac{4 a_{\mathrm{r}}^{\prime}-b_{\mathrm{r}}^{\prime}+\Delta-12 \mathrm{i} e_{\mathrm{r}}^{\prime}}{t} & \pm \frac{1}{\sqrt{3}} \frac{4 a_{\mathrm{r}}^{\prime}-b_{\mathrm{r}}^{\prime}+\Delta-6 \mathrm{i} e_{\mathrm{r}}^{\prime}}{t} & -\frac{1}{\sqrt{2}} \\
\pm \frac{1}{\sqrt{6}} \frac{4 a_{\mathrm{r}}^{\prime}-b_{\mathrm{r}}^{\prime}+\Delta-12 \mathrm{i} e_{\mathrm{r}}^{\prime}}{t} & \pm \frac{1}{\sqrt{3}} \frac{4 a_{\mathrm{r}}^{\prime}-b_{\mathrm{r}}^{\prime}+\Delta-6 \mathrm{i} e_{\mathrm{r}}^{\prime}}{t} & \frac{1}{\sqrt{2}}
\end{array}\right)
$$

where $\Delta=\sqrt{\left(-4 a_{\mathrm{r}}^{\prime}+b_{\mathrm{r}}^{\prime}\right)^{2}+72 e_{\mathrm{r}}^{\prime 2}}$ and $t=\sqrt{\left(4 a_{\mathrm{r}}^{\prime}-b_{\mathrm{r}}^{\prime}+\Delta\right)^{2}+72 e_{\mathrm{r}}^{\prime 2}}$, and the " \pm " signs correspond to the sign of $e_{\mathrm{r}}^{\prime}$. It is obvious that $V$ can be regarded as a variation of the tri-bimaximal flavor mixing pattern, and the equalities $\left|V_{\mu i}\right|=\left|V_{\tau i}\right|$ hold (for $i=1,2,3$ ). To be explicit,

$$
\begin{aligned}
& \theta_{13}=0 \\
& \theta_{23}=\frac{\pi}{4}, \quad \theta_{12}=\arctan \left[\frac{1}{\sqrt{2}} \sqrt{\frac{\left(4 a_{\mathrm{r}}^{\prime}-b_{\mathrm{r}}^{\prime}+\Delta\right)^{2}+144 e_{\mathrm{r}}^{\prime 2}}{\left(4 a_{\mathrm{r}}^{\prime}-b_{\mathrm{r}}^{\prime}+\Delta\right)^{2}+36 e_{\mathrm{r}}^{\prime 2}}}\right], \\
& \delta \in[0,2 \pi), \\
& \rho=-\varphi_{2} \text { or }-\varphi_{2}+\pi, \quad \sigma=-\varphi_{1}-\pi \text { or }-\varphi_{1} \text {, } \\
& \phi_{e}=\varphi_{1}+\varphi_{2}+\pi, \quad \phi_{\mu}=\pi \\
& \phi_{\tau}=0 \text {. }
\end{aligned}
$$

where $\tan \varphi_{1}=6 e_{\mathrm{r}}^{\prime} /\left(4 a_{\mathrm{r}}^{\prime}-b_{\mathrm{r}}^{\prime}+\Delta\right)$ and $\tan \varphi_{2}=12 e_{\mathrm{r}}^{\prime} /\left(4 a_{\mathrm{r}}^{\prime}-b_{\mathrm{r}}^{\prime}+\Delta\right)$.

Given eq. (5.2), a straightforward calculation leads us to the conclusion $\epsilon_{1} \neq 0$ in the basis where $M_{\mathrm{R}}$ is diagonal (i.e., $M_{\mathrm{R}}=\widehat{M}_{N}=\operatorname{Diag}\left\{d_{\mathrm{r}}-e_{\mathrm{r}}-\Delta^{\prime}, d_{\mathrm{r}}-e_{\mathrm{r}}+\Delta^{\prime}, a_{\mathrm{r}}+b_{\mathrm{r}}+e_{\mathrm{r}}\right\}$ with $\left.\Delta^{\prime}=\sqrt{a_{\mathrm{r}}^{2}-a_{\mathrm{r}} b_{\mathrm{r}}+b_{\mathrm{r}}^{2}-2 a_{\mathrm{r}} d_{\mathrm{r}}+b_{\mathrm{r}} d_{\mathrm{r}}+d_{\mathrm{r}}^{2}+a_{\mathrm{r}} e_{\mathrm{r}}-2 b_{\mathrm{r}} e_{\mathrm{r}}-d_{\mathrm{r}} e_{\mathrm{r}}+e_{\mathrm{r}}^{2}}\right)$. It is therefore possible to realize both unflavored and flavored leptogenesis in this case.

Finally, let us make another remark. Although the $S_{3}$ reflection symmetry helps a lot in determining the flavor structures in the seesaw mechanism, it must be broken so as to make the relevant phenomenological results fit current experimental data to a good or acceptable degree of accuracy. A further work along this line of thought will be done somewhere else. All in all, we expect that our structural classification and discussions in the $S_{3}$ reflection symmetry limit will be useful for phenomenological studies of neutrino mass

\footnotetext{
${ }^{5}$ The normal hierarchy case will not be discussed here, because it yields $\theta_{12}=\pi / 2$ and thus disfavored.
} 
generation, lepton flavor mixing, CP violation and leptogenesis when specific symmetry breaking effects and more accurate experimental data are taken into account. The same idea and similar analyses can be extended and applied to some other seesaw mechanisms.

\section{Acknowledgments}

We would like to thank Shun Zhou and Jing-yu Zhu for useful discussions. This work is supported in part by the National Natural Science Foundation of China under Grant no. 11775231 and no. 11835013.

Open Access. This article is distributed under the terms of the Creative Commons Attribution License (CC-BY 4.0), which permits any use, distribution and reproduction in any medium, provided the original author(s) and source are credited.

\section{References}

[1] Particle Data Group collaboration, Review of particle physics, Phys. Rev. D 98 (2018) 030001 [INSPIRE].

[2] P. Minkowski, $\mu \rightarrow$ e $\gamma$ at a rate of one out of $10^{9}$ muon decays?, Phys. Lett. B 67 (1977) 421 [INSPIRE].

[3] T. Yanagida, Horizontal gauge symmetry and masses of neutrinos, in Proceedings of the Workshop on Unified Theory and the Baryon Number of the Universe, O. Sawada and A. Sugamoto eds., KEK, Tsukuba, Japan (1979), pg. 95 [INSPIRE].

[4] M. Gell-Mann, P. Ramond and R. Slansky, Complex spinors and unified theories, in Supergravity, P. van Nieuwenhuizen and D. Freedman eds., North Holland, Amsterdam, The Netherlands (1979), pg. 315 [Conf. Proc. C 790927 (1979) 315] [arXiv:1306.4669] [INSPIRE].

[5] S.L. Glashow, The future of elementary particle physics, in Quarks and leptons, M. Lévy et al. eds., Plenum, New York, U.S.A. (1980), pg. 707 [NATO Sci. Ser. B 61 (1980) 687] [INSPIRE].

[6] R.N. Mohapatra and G. Senjanović, Neutrino mass and spontaneous parity nonconservation, Phys. Rev. Lett. 44 (1980) 912 [INSPIRE].

[7] H. Fritzsch and Z.-Z. Xing, Mass and flavor mixing schemes of quarks and leptons, Prog. Part. Nucl. Phys. 45 (2000) 1 [hep-ph/9912358] [InSPIRE].

[8] G. Altarelli and F. Feruglio, Discrete flavor symmetries and models of neutrino mixing, Rev. Mod. Phys. 82 (2010) 2701 [arXiv:1002.0211] [INSPIRE].

[9] H. Ishimori, T. Kobayashi, H. Ohki, Y. Shimizu, H. Okada and M. Tanimoto, Non-Abelian discrete symmetries in particle physics, Prog. Theor. Phys. Suppl. 183 (2010) 1 [arXiv: 1003.3552] [INSPIRE].

[10] S.F. King, Neutrino mass models, Rept. Prog. Phys. 67 (2004) 107 [hep-ph/0310204] [INSPIRE].

[11] Z. Maki, M. Nakagawa and S. Sakata, Remarks on the unified model of elementary particles, Prog. Theor. Phys. 28 (1962) 870 [inSPIRE]. 
[12] B. Pontecorvo, Neutrino experiments and the problem of conservation of leptonic charge, Sov. Phys. JETP 26 (1968) 984 [Zh. Eksp. Teor. Fiz. 53 (1967) 1717] [InSPIRE].

[13] Z.-Z. Xing and Z.-H. Zhao, A review of $\mu-\tau$ flavor symmetry in neutrino physics, Rept. Prog. Phys. 79 (2016) 076201 [arXiv:1512.04207] [InSPIRE].

[14] T. Kobayashi, Y. Shimizu, K. Takagi, M. Tanimoto, T.H. Tatsuishi and H. Uchida, Finite modular subgroups for fermion mass matrices and baryon/lepton number violation, arXiv: 1812.11072 [INSPIRE].

[15] P.F. Harrison and W.G. Scott, $\mu-\tau$ reflection symmetry in lepton mixing and neutrino oscillations, Phys. Lett. B 547 (2002) 219 [hep-ph/0210197] [InSPIRE].

[16] M. Fukugita and T. Yanagida, Baryogenesis without grand unification, Phys. Lett. B 174 (1986) 45 [inSPIRE].

[17] S. Pakvasa and H. Sugawara, Discrete symmetry and Cabibbo angle, Phys. Lett. B 73 (1978) 61 [INSPIRE].

[18] H. Harari, H. Haut and J. Weyers, Quark masses and Cabibbo angles, Phys. Lett. B 78 (1978) 459 [INSPIRE].

[19] H. Fritzsch and Z.-Z. Xing, Lepton mass hierarchy and neutrino oscillations, Phys. Lett. B 372 (1996) 265 [hep-ph/9509389] [INSPIRE].

[20] H. Fritzsch and Z.-Z. Xing, Large leptonic flavor mixing and the mass spectrum of leptons, Phys. Lett. B 440 (1998) 313 [hep-ph/9808272] [InSPIRE].

[21] M. Fukugita, M. Tanimoto and T. Yanagida, Atmospheric neutrino oscillation and a phenomenological lepton mass matrix, Phys. Rev. D 57 (1998) 4429 [hep-ph/9709388] [INSPIRE].

[22] W. Rodejohann and Z.-Z. Xing, Flavor democracy and type-II seesaw realization of bilarge neutrino mixing, Phys. Lett. B 601 (2004) 176 [hep-ph/0408195] [INSPIRE].

[23] J.E. Kim and J.-C. Park, Quantum numbers of heavy neutrinos, tri-bi-maximal mixing through double seesaw with permutation symmetry and comment on $\theta_{\text {sol }}+\theta_{c} \simeq \frac{\pi}{4}$, JHEP 05 (2006) 017 [hep-ph/0512130] [INSPIRE].

[24] R.N. Mohapatra, S. Nasri and H.-B. Yu, $S_{3}$ symmetry and tri-bimaximal mixing, Phys. Lett. B 639 (2006) 318 [hep-ph/0605020] [INSPIRE].

[25] Z.-Z. Xing, D. Yang and S. Zhou, Broken $S_{3}$ flavor symmetry of leptons and quarks: mass spectra and flavor mixing patterns, Phys. Lett. B 690 (2010) 304 [arXiv:1004.4234] [INSPIRE].

[26] E. Ma and R. Srivastava, Dirac or inverse seesaw neutrino masses with B - L gauge symmetry and $S_{3}$ flavor symmetry, Phys. Lett. B 741 (2015) 217 [arXiv:1411.5042] [INSPIRE].

[27] J.C. Gómez-Izquierdo, Non-minimal flavored $S_{3} \otimes Z_{2}$ left-right symmetric model, Eur. Phys. J. C 77 (2017) 551 [arXiv:1701.01747] [INSPIRE].

[28] Z.-G. Si, X.-H. Yang and S. Zhou, Broken $S_{3 \mathrm{~L}} \times S_{3 \mathrm{R}}$ flavor symmetry and leptonic CP-violation, Chin. Phys. C 41 (2017) 113105 [arXiv:1706.03991] [INSPIRE].

[29] J.C. Gómez-Izquierdo and M. Mondragón, $B-L$ model with $S_{3}$ symmetry: nearest neighbor interaction textures and broken $\mu \leftrightarrow \tau$ symmetry, arXiv:1804.08746 [INSPIRE]. 
[30] E.A. Garcés, J.C. Gómez-Izquierdo and F. Gonzalez-Canales, Flavored non-minimal left-right symmetric model fermion masses and mixings, Eur. Phys. J. C 78 (2018) 812 [arXiv: 1807.02727] [INSPIRE].

[31] M. Tanimoto, Vacuum neutrino oscillations of solar neutrinos and lepton mass matrices, Phys. Rev. D 59 (1999) 017304 [hep-ph/9807283] [INSPIRE].

[32] M. Tanimoto, Large mixing angle $M S W$ solution in $S_{3}$ flavor symmetry, Phys. Lett. B 483 (2000) 417 [hep-ph/0001306] [INSPIRE].

[33] G.C. Branco and J.I. Silva-Marcos, The symmetry behind extended flavor democracy and large leptonic mixing, Phys. Lett. B 526 (2002) 104 [hep-ph/0106125] [INSPIRE].

[34] P.F. Harrison and W.G. Scott, Permutation symmetry, tri-bimaximal neutrino mixing and the $S_{3}$ group characters, Phys. Lett. B 557 (2003) 76 [hep-ph/0302025] [InSPIRE].

[35] R. Jora, J. Schechter and M. Naeem Shahid, Perturbed $S_{3}$ neutrinos, Phys. Rev. D 80 (2009) 093007 [Erratum ibid. D 82 (2010) 079902] [arXiv:0909.4414] [INSPIRE].

[36] R. Jora, J. Schechter and M.N. Shahid, Doubly perturbed $S_{3}$ neutrinos and the $s_{13}$ mixing parameter, Phys. Rev. D 82 (2010) 053006 [arXiv:1006.3307] [INSPIRE].

[37] R. Jora, J. Schechter and M.N. Shahid, Naturally perturbed $S_{3}$ neutrinos, Int. J. Mod. Phys. A 28 (2013) 1350028 [arXiv:1210.6755] [INSPIRE].

[38] S. Dev, S. Gupta and R.R. Gautam, Broken $S_{3}$ symmetry in the neutrino mass matrix, Phys. Lett. B 702 (2011) 28 [arXiv:1106.3873] [INSPIRE].

[39] S. Dev, R.R. Gautam and L. Singh, Broken $S_{3}$ symmetry in the neutrino mass matrix and non-zero $\theta_{13}$, Phys. Lett. B 708 (2012) 284 [arXiv:1201.3755] [INSPIRE].

[40] H.B. Benaoum, Broken $S_{3}$ neutrinos, Phys. Rev. D 87 (2013) 073010 [arXiv:1302.0950] [INSPIRE].

[41] R. Barbieri, P. Creminelli, A. Strumia and N. Tetradis, Baryogenesis through leptogenesis, Nucl. Phys. B 575 (2000) 61 [hep-ph/9911315] [INSPIRE].

[42] T. Endoh, T. Morozumi and Z.-H. Xiong, Primordial lepton family asymmetries in seesaw model, Prog. Theor. Phys. 111 (2004) 123 [hep-ph/0308276] [INSPIRE].

[43] G.F. Giudice, A. Notari, M. Raidal, A. Riotto and A. Strumia, Towards a complete theory of thermal leptogenesis in the SM and MSSM, Nucl. Phys. B 685 (2004) 89 [hep-ph/0310123] [INSPIRE].

[44] T. Fukuyama and H. Nishiura, Mass matrix of Majorana neutrinos, hep-ph/9702253 [INSPIRE].

[45] E. Ma and M. Raidal, Neutrino mass, muon anomalous magnetic moment and lepton flavor nonconservation, Phys. Rev. Lett. 87 (2001) 011802 [Erratum ibid. 87 (2001) 159901] [hep-ph/0102255] [INSPIRE].

[46] C.S. Lam, A $2-3$ symmetry in neutrino oscillations, Phys. Lett. B 507 (2001) 214 [hep-ph/0104116] [INSPIRE].

[47] K.R.S. Balaji, W. Grimus and T. Schwetz, The solar LMA neutrino oscillation solution in the Zee model, Phys. Lett. B 508 (2001) 301 [hep-ph/0104035] [InSPIRE]. 
[48] I. Esteban, M.C. Gonzalez-Garcia, A. Hernandez-Cabezudo, M. Maltoni and T. Schwetz, Global analysis of three-flavour neutrino oscillations: synergies and tensions in the determination of $\theta_{23}, \delta_{\mathrm{CP}}$ and the mass ordering, JHEP 01 (2019) 106 [arXiv:1811.05487] [INSPIRE].

[49] P.F. Harrison, D.H. Perkins and W.G. Scott, Tri-bimaximal mixing and the neutrino oscillation data, Phys. Lett. B 530 (2002) 167 [hep-ph/0202074] [INSPIRE].

[50] Z.-Z. Xing, Nearly tri bimaximal neutrino mixing and CP-violation, Phys. Lett. B 533 (2002) 85 [hep-ph/0204049] [INSPIRE].

[51] X.G. He and A. Zee, Some simple mixing and mass matrices for neutrinos, Phys. Lett. B 560 (2003) 87 [hep-ph/0301092] [INSPIRE].

[52] S. Davidson, E. Nardi and Y. Nir, Leptogenesis, Phys. Rept. 466 (2008) 105 [arXiv:0802.2962] [INSPIRE].

[53] A.D. Sakharov, Violation of CP invariance, $C$ asymmetry and baryon asymmetry of the universe, Pisma Zh. Eksp. Teor. Fiz. 5 (1967) 32 [JETP Lett. 5 (1967) 24] [Sov. Phys. Usp. 34 (1991) 392] [Usp. Fiz. Nauk 161 (1991) 61] [INSPIRE].

[54] F.R. Klinkhamer and N.S. Manton, A saddle point solution in the Weinberg-Salam theory, Phys. Rev. D 30 (1984) 2212 [inSPIRE].

[55] W. Buchmüller, R.D. Peccei and T. Yanagida, Leptogenesis as the origin of matter, Ann. Rev. Nucl. Part. Sci. 55 (2005) 311 [hep-ph/0502169] [INSPIRE].

[56] Z.Z. Xing and S. Zhou, Neutrinos in particle physics, astronomy and cosmology, Zhejiang University Press and Springer-Verlag, Berlin Heidelberg, Germany (2011) [INSPIRE].

[57] E. Nardi, Y. Nir, E. Roulet and J. Racker, The importance of flavor in leptogenesis, JHEP 01 (2006) 164 [hep-ph/0601084] [INSPIRE].

[58] A. Abada, S. Davidson, F.-X. Josse-Michaux, M. Losada and A. Riotto, Flavor issues in leptogenesis, JCAP 04 (2006) 004 [hep-ph/0601083] [INSPIRE].

[59] G. Altarelli and F. Feruglio, Tri-bimaximal neutrino mixing, $A_{4}$ and the modular symmetry, Nucl. Phys. B 741 (2006) 215 [hep-ph/0512103] [INSPIRE].

[60] E. Ma and G. Rajasekaran, Softly broken $A_{4}$ symmetry for nearly degenerate neutrino masses, Phys. Rev. D 64 (2001) 113012 [hep-ph/0106291] [INSPIRE].

[61] K.S. Babu and X.-G. He, Model of geometric neutrino mixing, hep-ph/0507217 [INSPIRE]. 